Research Article

\title{
On Some Fundamental Peculiarities of the Traveling Wave Reactor
}

\author{
V. D. Rusov, ${ }^{1}$ V. A. Tarasov, ${ }^{1}$ I. V. Sharph,${ }^{1}$ V. N. Vashchenko, ${ }^{2}$ E. P. Linnik, ${ }^{1}$ \\ T. N. Zelentsova, ${ }^{1}$ M. E. Beglaryan, ${ }^{1}$ S. A. Chernegenko, ${ }^{1}$ S. I. Kosenko, ${ }^{1}$ and V. P. Smolyar ${ }^{1}$ \\ ${ }^{1}$ Odessa National Polytechnic University, Shevchenko Avenue 1, Odessa 65044, Ukraine \\ ${ }^{2}$ State Ecological Academy for Postgraduate Education and Management, 35 Mytropolyt Vasyl Lypkivskyi Street, Kyiv 03035, Ukraine
}

Correspondence should be addressed to V. D. Rusov; siiis@te.net.ua

Received 24 October 2014; Accepted 5 February 2015

Academic Editor: Michael I. Ojovan

Copyright (C) 2015 V. D. Rusov et al. This is an open access article distributed under the Creative Commons Attribution License, which permits unrestricted use, distribution, and reproduction in any medium, provided the original work is properly cited.

On the basis of the condition for nuclear burning wave existence in the neutron-multiplying media ( $\mathrm{U}-\mathrm{Pu}$ and Th- $\mathrm{U}$ cycles) we show the possibility of surmounting the so-called dpa-parameter problem and suggest an algorithm of the optimal nuclear burning wave mode adjustment, which is supposed to yield the wave parameters (fluence/neutron flux, width and speed of nuclear burning wave) that satisfy the dpa-condition associated with the tolerable level of the reactor materials radioactive stability, in particular that of the cladding materials. It is shown for the first time that the capture and fission cross sections of ${ }^{238} \mathrm{U}$ and ${ }^{239} \mathrm{Pu}$ increase with temperature within 1000-3000 K range, which under certain conditions may lead to a global loss of the nuclear burning wave stability. Some variants of the possible stability loss due to the so-called blow-up modes (anomalous nuclear fuel temperature and neutron flow evolution) are discussed and are found to possibly become a reason for a trivial violation of the traveling wave reactor internal safety.

\section{Introduction}

Despite the obvious and unique effectiveness of nuclear energy of the new generation, there are difficulties of its understanding related to the nontrivial properties of an ideal nuclear reactor of the future.

First, nuclear fuel should be natural, that is, nonenriched uranium or thorium. Second, traditional control rods should be absolutely absent in reactor active zone control system. Third, despite the absence of the control rods, the reactor must exhibit the so-called internal safety. This means that under any circumstances the reactor active zone must stay at a critical state, that is, sustain a normal operation mode automatically, with no operator actions, through physical causes and laws that naturally prevent the explosion-type chain reaction. Figuratively speaking, the reactors with internal safety are "the nuclear devices that never explode" [1].

Surprisingly, reactors that meet such unusual requirements are really possible. The idea of such self-regulating fast reactor was expressed for the first time in a general form (the so-called breed-and-burn mode) by Russian physicists Feynberg and Kunegin during the II Geneva conference in 1958 [2] and was relatively recently "reanimated" in a form of the self-regulating fast reactor in traveling nuclear burning wave mode by Russian physicist Feoktistov [3] and independently by American physicists Teller et al. [4].

The main idea of the reactor with internal safety is that the fuel components are chosen in such a way that, first, the characteristic time $\tau_{\beta}$ of the active fuel component (the fissile component) nuclear burning is significantly larger than the time of the delayed neutrons appearance, and, second, all the self-regulation conditions are sustained in the operation mode. Particularly, the equilibrium concentration $\widetilde{n}_{\text {fis }}$ of the active fuel component, according to Feoktistov's condition of the wave mode existence, is greater than its critical concentration (concentrations of the active element $\left({ }^{239} \mathrm{Pu}\right.$ and ${ }^{233} \mathrm{U}$ in cycles (1) and (2)) are called equilibrium or critical when an equal number of the active element nuclei 
or neutrons, respectively, is born and destroyed at the same time during the nuclear cycle) $n_{\text {crit }}$ [3]. These conditions are very important, though they are almost always practically implementable in case when the nuclear transformations chain of Feoktistov's uranium-plutonium cycle type [3] is significant among other reactions in the reactor:

$$
{ }^{238} \mathrm{U}(n, \gamma) \longrightarrow{ }^{239} \mathrm{U} \stackrel{\beta^{-}}{\longrightarrow}{ }^{239} \mathrm{~Np} \stackrel{\beta^{-}}{\longrightarrow}{ }^{239} \mathrm{Pu}(n, \text { fission }) .
$$

The same is also true for the Teller-Ishikawa-Wood thorium-uranium cycle type [4]:

$$
{ }^{232} \mathrm{Th}(n, \gamma) \longrightarrow{ }^{233} \mathrm{Th} \stackrel{\beta^{-}}{\longrightarrow}{ }^{233} \mathrm{~Pa} \stackrel{\beta^{-}}{\longrightarrow}{ }^{233} \mathrm{U}(n, \text { fission })
$$

In these cases the fissionable isotopes form ${ }^{239} \mathrm{Pu}$ in (1) or ${ }^{233} \mathrm{U}$ in (2)) which are the active components of the nuclear fuel. The characteristic time of such reaction depends on the time of the corresponding $\beta$-decays and is approximately equal to $\tau_{\beta}=2.3 / \ln 2 \approx 3.3$ days in case (1) and $\tau_{\beta} \approx 39.5$ days in case (2) which is many orders of magnitude higher than the corresponding time for the delayed neutrons.

The effect of the nuclear burning process self-regulation is provided by the fact that the system, being left by itself, cannot surpass the critical state and enter the uncontrolled reactor runaway mode, because the critical concentration is limited from above by a finite value of the active fuel component equilibrium concentration (plutonium in (1) or uranium in (2)): $\widetilde{n}_{\text {fis }}>n_{\text {crit }}$ (the Feoktistov's wave existence condition [3]).

Phenomenologically the process of the nuclear burning self-regulation is as follows. Any increase in neutron flow leads to a quick burnout of the active fuel component (plutonium in (1) or uranium in (2)), that is, to a reduction of their concentration and neutron flow; meanwhile, the formation of the new nuclei by the corresponding active fuel component proceeds with the prior rate during the time $\tau_{\beta}$. On the other hand, if the neutron flow drops due to some external impact, the burnout speed reduces and the active component nuclei generation rate increases, followed by the increase of a number of neutrons generated in the reactor during approximately the same time $\tau_{\beta}$.

The system of kinetic equations for nuclei (the components of nuclear fuel) and neutrons (in diffuse approximation) in such chains is rather simple. They differ only by the depth of description of all the possible active fuel components and nonburnable poison (here by poison we mean the oxygen nuclei or other elements, chemically bound to heavy nuclides, construction materials, coolant, and the poison itself, that is, the nuclei added to the initial reactor composition in order to control the neutron balance). Figure 1 shows the characteristic solutions for such problem (equations (3)-(9) in [5]) in a form of the soliton-like waves of the nuclear fuel components and neutrons concentrations for uraniumplutonium cycle in a cylindrical geometry case. Within the theory of the soliton-like fast reactors it is easy to show that in general case the phase speed $u$ of soliton-like neutron wave of nuclear burning is defined by the following approximate equality [5]:

$$
\begin{gathered}
\frac{u \tau_{\beta}}{2 L} \simeq\left(\frac{8}{3 \sqrt{\pi}}\right)^{6} \exp \left(-\frac{64}{9 \pi} a^{2}\right), \\
a^{2}=\frac{\pi^{2}}{4} \cdot \frac{n_{\text {crit }}}{\widetilde{n}_{\text {fis }}-n_{\text {crit }}},
\end{gathered}
$$

where $\widetilde{n}_{\text {fis }}$ and $n_{\text {crit }}$ are the equilibrium and critical concentration of the active (fissile) isotope, $L$ is the mean neutron diffusion length, and $\tau_{\beta}$ is the delay time, associated with the birth of the active (fissile) isotope and equal to an effective $\beta$-decay period of the intermediate nuclei in Feoktistov's uranium-plutonium cycle (1) or in Teller-Ishikawa-Wood thorium-uranium cycle (2).

Let us note that expression (3) automatically incorporates a condition of nuclear burning process self-regulation, since the fact of a wave existence is obviously predetermined by the inequality $\tilde{n}_{\text {fis }}>n_{\text {crit }}$. In other words, the expression (3) is a necessary physical condition of the soliton-like neutron wave existence. Let us note for comparison that the maximal value of the nuclear burning wave phase velocity, as follows from (3), is characterized for both uranium and thorium cycles by the equal average diffusion length $(L \sim 5 \mathrm{~cm})$ of the fast neutrons $(1 \mathrm{MeV})$ and is equal to $3.70 \mathrm{~cm} /$ day for uraniumplutonium cycle (4) and $0.31 \mathrm{~cm} /$ day for thorium-uranium cycle (2).

Generalizing the results of a wide range of numerical experiments [5-23], we can positively affirm that the principal possibility of the main stationary wave parameters control was reliably established within the theory of a self-regulating fast reactor in traveling wave mode, or, in other words, the traveling wave reactor (TWR). It is possible both to increase the speed, the thermal power, and the final fluence and to decrease them. Obviously, according to (3), it is achieved by varying the equilibrium and critical concentrations of the active fuel component, that is, by the purposeful change of the initial nuclear fuel composition.

The technological problems of TWR are actively discussed in science nowadays. The essence of these problems usually comes to a principal impossibility of such project realization and is defined by the following insurmountable flaws:

(i) high degree of nuclear fuel burnup (over 20\% in average) leading to the following adverse consequences:

(a) high damaging dose of fast neutrons acting at at the constructional materials ( $\sim 500 \mathrm{dpa})$ (for comparison, the claimed parameters for the Russian FN-800 reactor are 93 dpa; at the same time, it is known that one of the main tasks of the construction materials radioactive stability investigations conducted at the Bochvar Hi-tech Institute for nonorganic materials (Moscow) is to achieve 133 to 164 dpa by 2020 !),

(b) high gas release, which requires an increased gas cavity length on top of a long fuel rod as it is, 

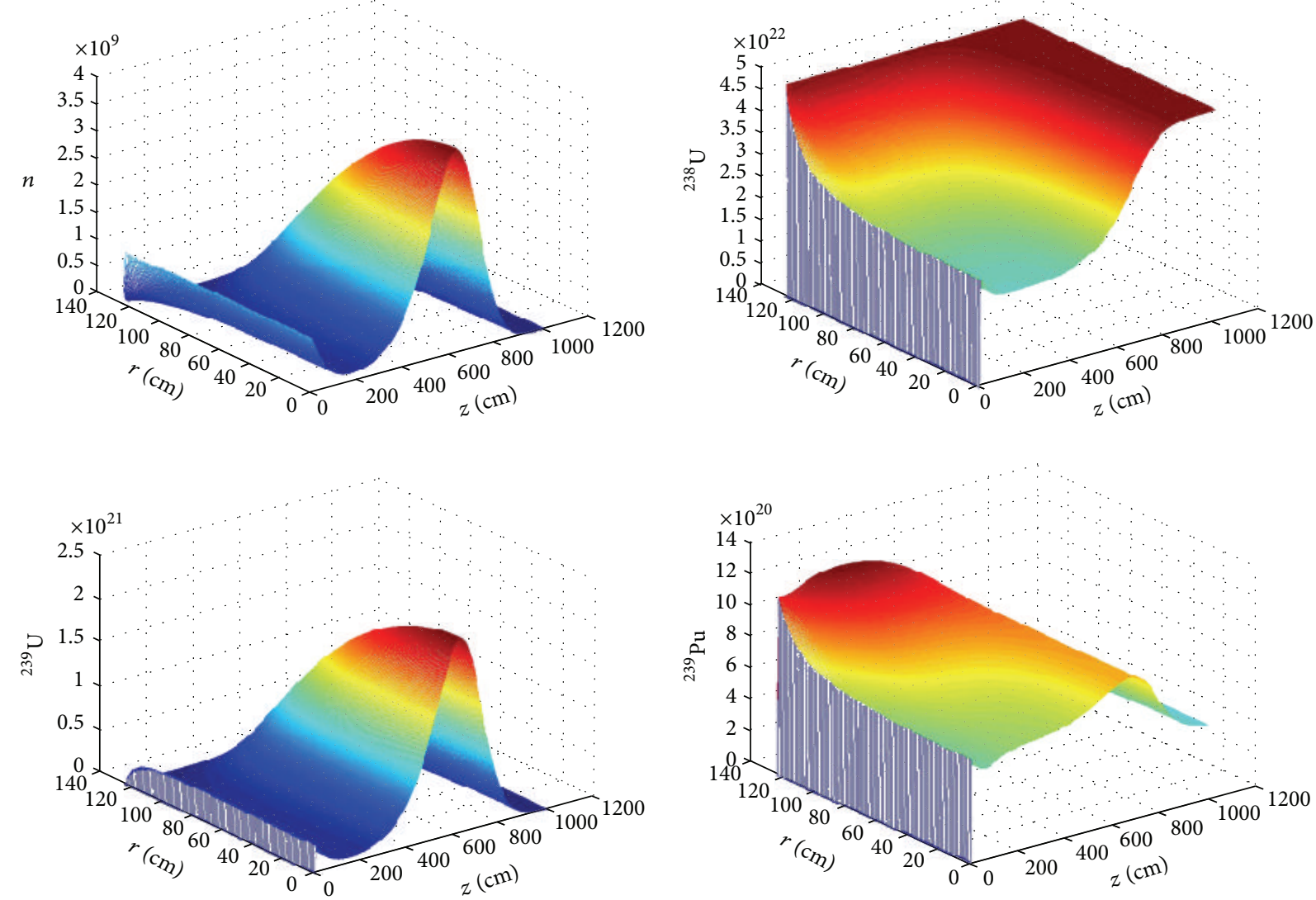

FIGURE 1: Kinetics of the neutrons ${ }^{238} \mathrm{U},{ }^{239} \mathrm{U}$, and ${ }^{239} \mathrm{Pu}$ concentrations in the core of a cylindrical reactor with radius of $125 \mathrm{~cm}$ and $1000 \mathrm{~cm}$ long at the time of 240 days. Here $r$ is the transverse spatial coordinate axis (cylinder radius); $z$ is the longitudinal spatial coordinate axis (cylinder length). Temporal step of the numerical calculations is $0.1 \mathrm{~s}$ adopted from [5].

(ii) long active zone requiring the correspondingly long fuel rods, which makes their parameters unacceptable from the technological use point of view and, for instance, this is the case for the parameters characterized by a significant increase in:

(a) the value of a positive void coefficient of reactivity,

(b) hydraulic resistance,

(c) energy consumption for the coolant circulation through the reactor,

(iii) the problem of nuclear waste associated with the unburned plutonium reprocessing and nuclear waste utilization.

The main goal of the present paper is to solve the specified technological problems of the TWR on the basis of a technical concept which makes it impossible for the damaging dose of the fast neutrons in the reactor (fuel rods jackets, reflection shield, and reactor pit) to exceed the $\sim 200 \mathrm{dpa}$ level. The essence of this technical concept is to provide the given neutron flux on in-reactor devices by defining the speed of the fuel movement relative to the nuclear burning wave speed. The neutron flux, wave speed, and fuel movement speed are in their turn predetermined by the chosen parameters (equilibrium and critical concentrations of the active component in the initial nuclear fuel composition).

Section 1 of this paper is dedicated to a brief analysis of the state-of-the-art idea of a self-regulating fast reactor in traveling wave mode. Based on this analysis we formulate the problem statement and chalk out the possible ways to solve it. Section 2 considers the analytical solution for a nonstationary $1 \mathrm{D}$ reactor equation in one-group approximation with negative reactivity feedback (1D van Dam [8] model). It yields the expressions for the amplitude $\varphi_{m}$, phase $\alpha$, and phase speed $u$, as well as the dispersion (FWHM) of the soliton-like burning wave. Knowing the FWHM we may further estimate the spatial distribution of the neutron flux and thus a final neutron fluence. Section 3 is dedicated to a description of the nontrivial neutron fluence dependence on phase velocity of the solitary burnup waves in case of the fissible and nonfissible absorbents. It reveals a possibility of the purposeful (in terms of the required neutron fluence and nuclear burning wave speed values) variation of the initial nuclear fuel composition. Section 4 analyzes the dependence of the damaging dose on neutron fluence, phase velocity, and dispersion of the solitary burnup waves. Section 5 considers the possible causes of the TWR internal safety violation caused by "Fukushima plutonium" effect or, in 
other words, the temperature blowup modes driven either by temperature or neutron flux. Section 6 is dedicated to analysis of the practical examples of the temperature blowup modes in neutron-multiplying media. The idea of an impulse thermonuclear TWR is also proposed. The conclusion of the paper is presented in Section 7.

\section{On Entropy and Dispersion of Solitary Burnup Waves}

Let us discuss the physical causes, defining the main characteristics of the soliton-like propagation of "criticality" wave in the initially undercritical environment, characterized by the infinite multiplication factor $k_{\infty}$ less than unit. Obviously, the supercritical area $\left(k_{\infty}>1\right)$ must be created by some external neutron source (e.g., by an accelerator or another supercritical area). In the general case, the supercritical area is a result of the breeding effects in fast nuclear systems or the burning of the fissible absorbents (fuel components) in thermal nuclear systems (Seifritz (1995) was the first to find theoretically a nuclear solitary burnup wave in opaque neutron absorbers [24]; the supercriticality waves in thermal nuclear reactors are investigated and analysed in the papers by Akhiezer et al. [25-28], where they show the possibility of both fast [25-27] and slow [28] modes of nuclear burning distribution (i.e., the supercriticality waves) in the framework of diffuse approximation.). Due to the gradual burn-out of the neutronmultiplying medium in the supercritical area, this area looses its supercritical properties, since $k_{\infty}$ becomes less than unit. The wave would have to stop and diminish at this point in an ordinary case. However, because of the neutrons, appearing during breeding and diffusely "infecting" the nearby areas, this "virgin" area before the wavefront is forced to obtain the properties of supercriticality, and the wave moves forward in this direction. Apparently, the stable movement of such soliton-like wave requires some kind of stabilizing mechanism, for example, the negative self-catalysis or any other negative feedback. This is called the negative reactivity feedback in traditional nuclear reactors (according to van Dam [8], the procedure of the reactivity introduction into the $1 \mathrm{D}$ nonstationary equation of the reactor in one-group approximation, though implicitly, takes the kinetic equations of the burnout into account, particularly, the production of plutonium in $\mathrm{U}-\mathrm{Pu}$ cycle or uranium in Th-U cycle). Let us therefore consider such an example qualitatively which is mentioned below.

For this purpose let us write down a 1D nonstationary equation of the reactor in one-group approximation [29-31] with negative reactivity feedback:

$$
D \frac{\partial^{2} \varphi}{\partial x^{2}}+\left(k_{\infty}-1+\gamma \varphi\right) \Sigma_{a} \varphi=\frac{1}{v} \frac{\partial \varphi}{\partial t},
$$

where $\varphi$ is the neutron flux $\left[\mathrm{cm}^{-2} \mathrm{~s}^{-1}\right], D$ is the diffusion coefficient $[\mathrm{cm}], \gamma \varphi$ is the reactivity, dimensionless value, $\Sigma_{a}$ is the total macroscopic absorption cross section $\left[\mathrm{cm}^{-1}\right]$, and $v$ is the neutron speed $\left[\mathrm{cm} \cdot \mathrm{s}^{-1}\right]$. In this case the negative feedback $\gamma$ is defined mainly by the fact that the infinite multiplication factor is used in (4), and therefore the flux density must be corrected.

Let us search for the solution in an autowave form:

$$
\varphi(x, t)=\varphi(x-u t) \equiv \varphi(\xi),
$$

where $u$ is the wave phase velocity and $\xi$ is the coordinate in a coordinate system, which moves with phase speed. In such case consider the following:

$$
\frac{1}{v} \frac{\partial \varphi}{\partial t}=-\frac{u}{v} \frac{\partial \varphi}{\partial t}
$$

As is known [8], the relation $u / v$ by order of magnitude is equal to $10^{-13}$ and $10^{-11}$ for fast and thermal nuclear systems, respectively. Therefore the partial time derivative in (4) may be neglected without loss of generality. Further taking into account (5), equation (4) may be presented in the following form:

$$
L^{2} \frac{\partial^{2} \varphi}{\partial \xi^{2}}+\left[k_{\infty}(\psi)-1+\gamma \varphi\right] \varphi=0,
$$

where $L=\left(D / \Sigma_{a}\right)^{1 / 2}$ is the neutron diffusion length, and $\psi$ is the so-called neutron fluence function:

$$
\psi(x, t)=\int_{0}^{t} \varphi\left(x, t^{\prime}\right) d t^{\prime} .
$$

In order to find a physically sensible analytic solution of (7) by substituting (8), we need to define some realistic form of the function $k_{\infty}(\psi)$ (usually referred to as the burnup function). Since the real burnup function $k_{\infty}(\psi)$ has a form of some asymmetric bell-shaped dependence on fluence, normalized to its maximal value $\psi_{\max }$ (Figure 1), following [8], let us define it in a form of parabolic dependency without losing generality:

$$
k_{\infty}=k_{\max }+\left(k_{0}-k_{\max }\right)\left(\frac{\psi}{\psi_{\max }}-1\right)^{2},
$$

where $k_{\max }$ and $k_{0}$ are the maximal and initial neutron multiplication factors. Substituting (9) into (7) we obtain the following:

$$
L^{2} \varphi_{\xi \xi}+\rho_{\max } \varphi+\gamma_{0} \varphi^{2}-\delta\left[\frac{\int_{\xi}^{\infty} \varphi d \xi}{u \psi_{m}}-1\right]^{2} \varphi=0,
$$

where $\rho_{\max }=k_{\max }-1, \delta=k_{\max }-k_{0}$, and $\gamma_{0} \equiv \gamma$.

Suppose we are searching for a partial solution of (10). Let us rewrite it in the following form:

$$
L^{2} \frac{d^{2} \varphi}{d \xi^{2}}+\left(\rho_{\max }+\gamma_{0} \varphi-\delta\left(\frac{\int_{\xi}^{+\infty} \varphi d \xi}{u \psi_{m}}-1\right)^{2}\right) \varphi=0 .
$$

Let us introduce a new unknown function,

$$
\chi(\xi)=\int_{\xi}^{+\infty} \varphi d \xi \Longrightarrow \varphi(\xi)=-\frac{d \chi(\xi)}{d \xi},
$$


that, due to its nonnegativity on the interval $\xi \in[0, \infty]$, must satisfy the following boundary conditions: $\varphi=0$ for $\xi=0, \infty$.

The equation will take the form

$$
\begin{aligned}
L^{2} \frac{d^{3} \chi(\xi)}{d \xi^{3}} & +\left(\rho_{\max }-\gamma_{0} \frac{d \chi(\xi)}{d \xi}-\delta\left(\frac{\chi(\xi)}{u \psi_{m}}-1\right)^{2}\right) \\
\cdot \frac{d \chi(\xi)}{d \xi} & =0 .
\end{aligned}
$$

In order to find a partial solution of (13), we require the following additional condition to hold:

$$
\rho_{\max }-\gamma_{0} \frac{d \chi(\xi)}{d \xi}-\delta\left(\frac{\chi(\xi)}{u \psi_{m}}-1\right)^{2}=f(\chi(\xi)),
$$

where $f(\xi)$ is an arbitrary function, the exact form of which will be defined later. The condition (14) is chosen because it makes it possible to integrate (13). Really, if (14) is true, (5) takes the following form:

$$
L^{2} \frac{d^{3} \chi(\xi)}{d \xi^{3}}+f(\chi(\xi)) \frac{d \chi(\xi)}{d \xi}=0 .
$$

That allows us to reduce the order of the equation:

$$
L^{2} \frac{d^{2} \chi(\xi)}{d \xi^{2}}+F_{1}(\chi(\xi))=C
$$

Here $F_{1}(\chi)$ denotes a primitive of $f(\chi)$, and $C$ is an arbitrary integration constant. The order of (16) may be further reduced by multiplying both sides of the equation by $d \chi(\xi) / d \xi$ :

$$
\frac{L^{2}}{2}\left(\frac{d \chi(\xi)}{d \xi}\right)^{2}+F_{2}(\chi(\xi))-C \chi(\xi)=B .
$$

$F_{2}(\chi)$ here denotes a primitive of $F_{1}(\chi)$, that is, "the second primitive" of the function $f(\chi)$ introduced in (5), and $B$ is a new integration constant.

The obtained equation (17) is a separable equation and may be rewritten in the following form:

$$
d \xi= \pm \frac{d \chi}{\sqrt{\left(2 / L^{2}\right)\left(B-F_{2}(\chi(\xi))+C \chi(\xi)\right)}} .
$$

On the other hand, (14) may also be considered a separable equation relative to $\chi(\xi)$. Then, separating variables in (14) we obtain the following:

$$
d \xi=d \chi\left(\frac{\rho_{\max }}{\gamma_{0}}-\frac{\delta}{\gamma_{0}}\left(\frac{\chi(\xi)}{u \psi_{m}}-1\right)^{2}-\frac{1}{\gamma_{0}} f(\chi(\xi))\right)^{-1}
$$

Since (18) and (19) are for the same function $\chi(\xi)$, by comparing them, we derive that the following relation must hold:

$$
\begin{aligned}
& \pm \sqrt{\frac{2}{L^{2}}\left(B-F_{2}(\chi(\xi))+C \chi(\xi)\right)} \\
& =\frac{\rho_{\max }}{\gamma_{0}}-\frac{\delta}{\gamma_{0}}\left(\frac{\chi(\xi)}{u \psi_{m}}-1\right)^{2}-\frac{1}{\gamma_{0}} f(\chi(\xi)) .
\end{aligned}
$$

In order to simplify (18) and (19), let us choose $f(\chi)$ in a polynomial form of $\chi$. The order of this polynomial is $n$. Then $F_{2}(\xi)$, obtained by double integration of $f(\chi)$, is a polynomial of order $(n+2)$. Taking the square root, according to (20), should also lead to a polynomial of the order $n$. Therefore $n+$ $2=2 n \Rightarrow n=2$.

Consequently, the function $f(\chi)$ may only be a secondorder polynomial under the assumptions made above. Consider the following:

$$
f(\chi)=s_{2} \chi^{2}+s_{1} \chi+s_{0}
$$

where $s_{0}, s_{1}$, and $s_{2}$ are the polynomial coefficients.

Double integration of (21) leads to the following:

$$
F_{2}(\chi)=\frac{s_{2}}{12} \chi^{4}+\frac{s_{1}}{6} \chi^{3}+\frac{s_{0}}{2} \chi^{2}+c_{1} \chi+c_{2},
$$

where $c_{1}$ and $c_{2}$ are the integration constants.

Substituting (21) and (22) into (20) we get the following:

$$
\begin{aligned}
& \left(\frac{\rho_{\max }}{\gamma_{0}}-\frac{\delta}{\gamma_{0}}\left(\frac{\chi}{u \psi_{m}}-1\right)^{2}-\frac{1}{\gamma_{0}}\left(s_{2} \chi^{2}+s_{1} \chi+s_{0}\right)\right)^{2} \\
& =\frac{2}{L^{2}}\left(B-\left(\frac{s_{2}}{12} \chi^{4}+\frac{s_{1}}{6} \chi^{3}+\frac{s_{0}}{2} \chi^{2}+c_{1} \chi+c_{2}\right)+C \chi\right) .
\end{aligned}
$$

Further in (23) we set the coefficients at the same orders of $\chi$ equal the following:

$$
\begin{gathered}
\left(\frac{\delta}{\gamma_{0} u^{2} \psi_{m}^{2}}+\frac{s_{2}}{\gamma_{0}}\right)^{2}=-\frac{s_{2}}{6 L^{2}}, \\
-2\left(\frac{\delta}{\gamma_{0} u^{2} \psi_{m}^{2}}+\frac{s_{2}}{\gamma_{0}}\right)\left(\frac{2 \delta}{\gamma_{0} u \psi_{m}}-\frac{s_{1}}{\gamma_{0}}\right)=-\frac{s_{1}}{3 L^{2}}, \\
\left(\frac{2 \delta}{\gamma_{0} u \psi_{m}}-\frac{s_{1}}{\gamma_{0}}\right)^{2}-2\left(\frac{\delta}{\gamma_{0} u^{2} \psi_{m}^{2}}+\frac{s_{2}}{\gamma_{0}}\right) \\
\cdot\left(\frac{\rho_{\max }}{\gamma_{0}}-\frac{\delta}{\gamma_{0}}-\frac{s_{0}}{\gamma_{0}}\right)=-\frac{s_{0}}{L^{2}}, \\
2\left(\frac{2 \delta}{\gamma_{0} u \psi_{m}}-\frac{s_{1}}{\gamma_{0}}\right)\left(\frac{\rho_{\max }}{\gamma_{0}}-\frac{\delta}{\gamma_{0}}-\frac{s_{0}}{\gamma_{0}}\right) \\
=-\left(\frac{2 c_{1}}{L^{2}}+\frac{2 C}{L^{2}}\right), \\
\left(\frac{\rho_{\max }}{\gamma_{0}}-\frac{\delta}{\gamma_{0}}-\frac{s_{0}}{\gamma_{0}}\right)^{2}=\frac{2}{L^{2}} B-\frac{2 c_{2}}{L^{2}} .
\end{gathered}
$$

Note that the first three equations are enough to find the coefficients $s_{0}, s_{1}$, and $s_{2}$, and the remaining two equations may be satisfied with the appropriate constants $B, C, c_{1}$, and $c_{2}$ as follows:

$$
\begin{gathered}
s_{0}=\rho_{\max }-\delta, \\
s_{1}=\frac{2 \delta}{u \psi_{m}}-\frac{\gamma_{0}}{L} \sqrt{\delta-\rho_{\max }} \\
s_{2}=\frac{\delta \gamma_{0}}{3 L u \psi_{m} \sqrt{\delta-\rho_{\max }}}-\frac{\gamma_{0}^{2}}{6 L^{2}}-\frac{\delta}{u^{2} \psi_{m}^{2}} .
\end{gathered}
$$


After finding $s_{0}, s_{1}$, and $s_{2}$ from this system, we may consider (19) in more detail, which takes the form:

$$
\begin{aligned}
d \xi= & d \chi\left(-\left(\frac{\delta}{\gamma_{0} u^{2} \psi_{m}^{2}}+\frac{s_{2}}{\gamma_{0}}\right) \chi^{2}+\left(\frac{2 \delta}{\gamma_{0} u \psi_{m}}+\frac{s_{1}}{\gamma_{0}}\right) \chi\right. \\
& \left.+\left(\frac{\rho_{\max }}{\gamma_{0}}-\frac{\delta}{\gamma_{0}}-\frac{s_{0}}{\gamma_{0}}\right)\right)^{-1} .
\end{aligned}
$$

Solving this equation yields

$$
\frac{d \chi}{(\chi-K)^{2}-M^{2}}=-N d \xi
$$

where

$$
\begin{gathered}
K=\frac{\delta / \gamma_{0} u \psi_{m}-s_{1} / 2 \gamma_{0}}{\delta / \gamma_{0} u^{2} \psi_{m}^{2}+s_{2} / \gamma_{0}}, \\
M^{2}=\left(\frac{\delta / \gamma_{0} u \psi_{m}-s_{1} / 2 \gamma_{0}}{\delta / \gamma_{0} u^{2} \psi_{m}^{2}+s_{2} / \gamma_{0}}\right)+\frac{\left(\rho_{\max } / \gamma_{0}-\delta / \gamma_{0}-s_{0} / \gamma_{0}\right)}{\left(\delta / \gamma_{0} u^{2} \psi_{m}^{2}+s_{2} / \gamma_{0}\right)}, \\
N=\frac{\delta}{\gamma_{0} u^{2} \psi_{m}^{2}}+\frac{s_{2}}{\gamma_{0}}
\end{gathered}
$$

Let us introduce a new variable $\chi_{1}$ into (31) by substituting the following:

$$
\chi-K=M \chi_{1}, \quad d \chi=M d \chi_{1}
$$

Then (31) will take the following form:

$$
\frac{d \chi_{1}}{\left(\chi_{1}\right)^{2}-1}=-M N d \xi
$$

Hence

$$
\chi_{1}=-\tanh (M N \xi-D)
$$

where $D$ is the integration constant. Taking into account that $\chi-K=M \chi_{1}$,

$$
\chi=K-M \tanh (M N \xi-D)
$$

Considering (12), we obtain the soliton-like solution in the form

$$
\varphi(\xi)=M^{2} N \operatorname{sech}^{2}(M N \xi-D)
$$

Let us remember that together with introducing a new unknown function $\chi(\xi)$ (see (12)) we obtained an obvious condition for this function:

$$
\lim _{\xi \rightarrow \infty} \chi(\xi)=0
$$

Let us show that this condition eventually leads to an autowave existence condition. Obviously condition (38) along with (36),

$$
\lim _{\xi \rightarrow \infty} \chi(\xi)=\lim _{\xi \rightarrow \infty}[K-M \tanh (M N \xi-D)]=0,
$$

leads to

$$
K=M
$$

This relation lets us define the amplitude $\varphi_{m}$, phase $\alpha$, and phase velocity $u$ of the soliton-like wave:

$$
\begin{gathered}
\alpha=M N=\frac{\sqrt{\delta-\rho_{\max }}}{2 L}, \\
\varphi_{m}=M^{2} N=\frac{\delta-3 \rho_{\max }}{2 \gamma_{0}}=\frac{3 \rho_{\max }-\delta}{2\left|\gamma_{0}\right|}, \\
u=\frac{\varphi_{m}}{\alpha \psi_{m}} .
\end{gathered}
$$

From the condition of nonnegative width (41) and amplitude (42) of the nuclear burning wave, the following is the condition of $1 \mathrm{D}$ autowave existence, or the so-called "ignition condition" by van Dam [8]:

$$
3 \rho_{\max }-\delta=2 k_{\max }+k_{0}-3 \geqslant 0, \quad \text { where } 1-k_{0}>0 \text {. }
$$

It is noteworthy that the analogous results for a nonlinear one-group diffusion 1D-model (4) with explicit feedback and burnup effects were first obtained by van Dam [8]. The same results (see (41)-(43)) were obtained by Chen and Maschek [11] while investigating the $3 \mathrm{D}$-model by van Dam using the perturbation method. The only difference is that the value of neutron fluence associated with the maximum of burnup parameter $k_{\infty}$ was adapted to the transverse buckling (Figure 2). In other words, they considered the transverse geometric buckling mode as a basis for perturbation. Hence they introduced a geometric multiplication factor $k_{\mathrm{GB}}$ due to transverse buckling into two-dimensional equation (4), which led to a change in some initial parameters $\left(\rho_{\max }=\right.$ $k_{\max }-k_{\mathrm{GB}}, \delta=k_{\max }-k_{0}$ ) and, consequently, to a change in the conditions of the autowave existence in $3 \mathrm{D}$ case,

$$
3 \rho_{\max }-\delta=2 k_{\max }+k_{0}-3 k_{\mathrm{GB}} \geqslant 0, \quad \text { where } k_{\mathrm{GB}}-k_{0}>0,
$$

that in the case of $k_{\mathrm{GB}}=1$ is exactly the same as the so-called ignition condition by van Dam [8].

From the point of view of the more detailed Feoktistov model [3] analysis, thoroughly considered in [5] and related to a concept of the nuclear systems internal safety, the condition (45) is necessary, but not sufficient. On the other hand, it is an implicit form of the necessary condition of wave 


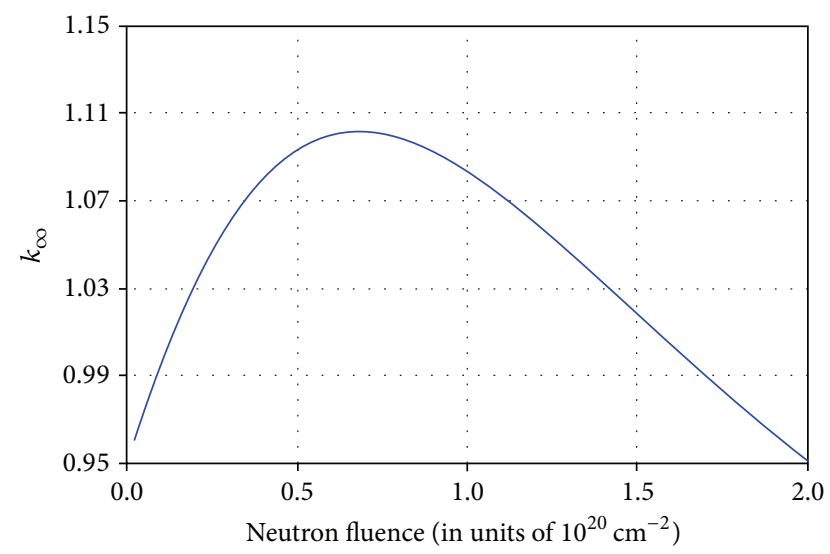

FIGURE 2: Asymmetric burnup function as characteristic for realistic burnup function, adapted from [8].

existence according to Feoktistov, where the equilibrium concentration $\tilde{n}_{\text {fis }}$ of the active fuel component must be greater than its critical concentration $n_{\text {crit }}\left(\widetilde{n}_{\text {fis }}>n_{\text {crit }}\right)$ [3, 5]. The physics of such hidden but simple relation will be explained below (see Section 3).

Returning to a $1 \mathrm{D}$ reactor equation solution (10) in onegroup approximation with negative reactivity feedback, let us write it in a more convenient form for analysis:

$$
\varphi=\varphi_{m} \operatorname{sech}^{2}(\alpha \xi)=\varphi_{m} \operatorname{sech}^{2}[\alpha(x-u t)]
$$

where $\varphi_{m}$ is the amplitude of the neutron flux and $1 / \alpha$ is the characteristic length proportional to the soliton wave width, which is a full width at half-maximum (FWHM) by definition and is equal to

$$
\Delta_{1 / 2}=\mathrm{FWHM}=2 \ln (1+\sqrt{2}) \alpha^{-1}[\mathrm{~cm}] .
$$

Apparently, integrating (45) yields the area under such soliton:

$$
A_{\text {area }}=\frac{2 \varphi_{m}}{\alpha}\left[\mathrm{cm}^{2}\right]
$$

In order to estimate the extent of the found parameters influence on the dynamics of the soliton-like nuclear burning wave stability, let us invoke an information-probability approach, developed by Seifritz [32]. For this purpose let us write down the expression for the mean value of information or, more precisely, the entropy of the studied process:

$$
S=-k_{B} \int_{-\infty}^{\infty} p(x) \ln p(x) d x
$$

where $p(x)$ is the function of probability density relative to a dimensionless variable $x ; \ln 1 / p(x)$ is the mean information value; $k_{B}$ is the Boltzmann constant.
Substituting the soliton-like solution (45) into (49) we obtain the following:

$$
\begin{aligned}
S & =-2 k_{B} \int_{0}^{\infty} \operatorname{sech}^{2}(\alpha \xi) \ln \left[\operatorname{sech}^{2}(\alpha \xi)\right] d\left(\frac{x}{u \tau_{\beta}}\right) \\
& =4 k_{B} \int_{0}^{\infty} \frac{\ln \cosh (\beta y)}{\cosh ^{2}(\beta y)} d y,
\end{aligned}
$$

where $\beta=\alpha\left(u \tau_{\beta}\right)$ is another (dimensionless) scaling factor and $\tau_{\beta}$ is the proper $\beta$-decay time of the active component of the nuclear fuel. Let us point out the procedure of making the $x$ argument dimensionless in (50), which takes into account the fact that the neutron flux amplitude is proportional to the phase velocity of the nuclear burning wave (see (43)), that is, $\varphi_{m} \sim u$. Calculating the integral (50) leads to the following quite simple expression for the entropy:

$$
\begin{aligned}
S & =\frac{4(1-\ln 2)}{\beta}=4(1-\ln 2) \frac{k_{B}}{\alpha u \tau_{\beta}} \\
& =\frac{4 k_{B}(1-\ln 2)}{\sqrt{k_{\mathrm{GB}}-k_{0}}} \frac{2 L}{u \tau_{\beta}},
\end{aligned}
$$

which in the case of

$$
S \sim k_{B} \frac{2 L}{u \tau_{\beta}}=\text { const, }
$$

points to an isoentropic transport of the nuclear burning wave.

It is interesting to note here that if the width $\Delta_{1 / 2} \rightarrow 0$, then due to isoentropicity of the process (52) the form of the soliton becomes similar to the so-called Dirac $\delta$-function. Introducing two characteristic sizes or two length scales $\left(l_{1}=\right.$ $1 / \alpha$ and $\left.l_{2}=\varphi_{m}\right)$, it is possible to see that when the first of them is small the second one increases and vice versa. It happens because the area $A_{\text {area }}$ (41) under the soliton must remain constant, since $A_{\text {area }} \propto l_{1} l_{2}$. In this case the soliton entropy tends to zero because the entropy is proportional to the ratio of these values $\left(S \propto l_{1} / l_{2} \rightarrow 0\right)$. These features are the consequence of the fact that the scale $l_{1}$ is a characteristic of a dispersion of the process, while another scale $l_{2}$ is a characteristic of the soliton nonlinearity. If $l_{1} \ll l_{2}$, then the process is weakly dispersed (Figure $3(\mathrm{a})$ ). If $l_{1} \gg l_{2}$, then the process is strongly dispersed (Figure 3(b)). In the latter case the soliton amplitude becomes relatively large (a case of $\delta$-function). And finally, if $l_{1}=1_{2}$, then the soliton speed $u \propto\left(l_{1}^{2} l_{2}^{2}\right)^{1 / 2}$ (see (44)) is proportional to the geometrical mean of the dispersion and nonlinearity parameters.

On the other hand, it is clear that, according to (41) and (43), the burning wave width $\Delta_{1 / 2}$ is a parameter that participates in formation of the time for neutron fluence accumulation $\tau_{\beta}$ on the internal surface of the TWR long fuel rod cladding material:

$$
\psi_{m} \sim \frac{\Delta_{1 / 2}}{u} \varphi_{m}=\tau_{\varphi} \cdot \varphi_{m}
$$




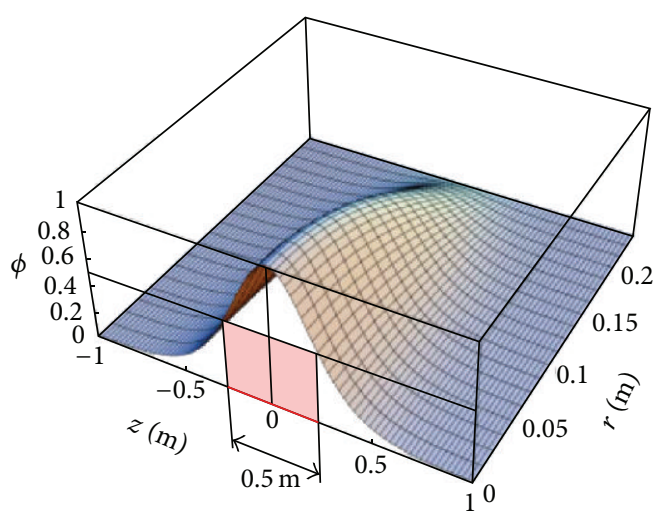

(a)

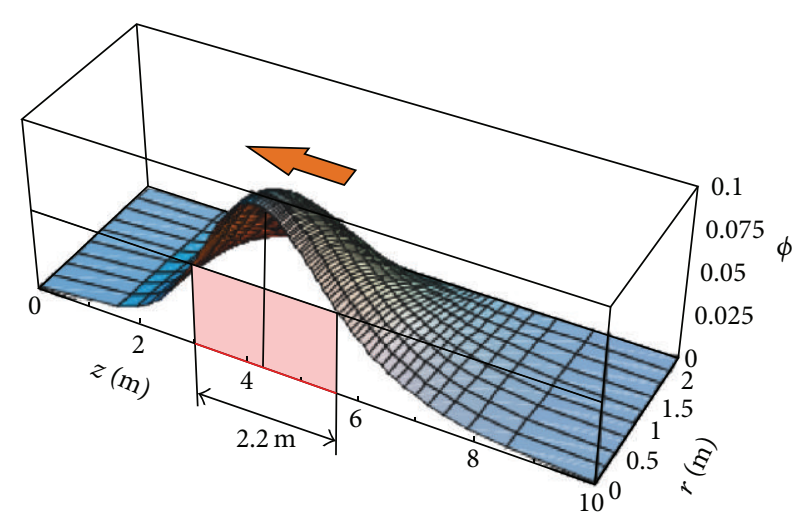

(b)

FIgURE 3: (a) Weakly dispersive wave pattern, obtained by Chen and Maschek [11], investigating a 3D-model by van Dam using perturbation method. Example with the following parameters: $L=0.02 \mathrm{~m}, k_{\mathrm{GB}}=1.04, k_{0}=1.02, k_{\max }=1.06, \phi_{m}=10^{17} \mathrm{~m}^{-2} \mathrm{~s}^{-1}$, and $u=0.244 \mathrm{~cm} / \mathrm{day}$. (b) Strongly dispersive wave pattern, obtained by Chen et al. [19] within a 3D-model of traveling wave reactor. Example with the following parameters: $L=0.017 \mathrm{~m}, k_{\mathrm{GB}}=1.00030, k_{0}=0.99955, \phi_{m}=3 \cdot 10^{15} \mathrm{~m}^{-2} \mathrm{~s}^{-1}$, and $u=0.05 \mathrm{~cm} /$ day.

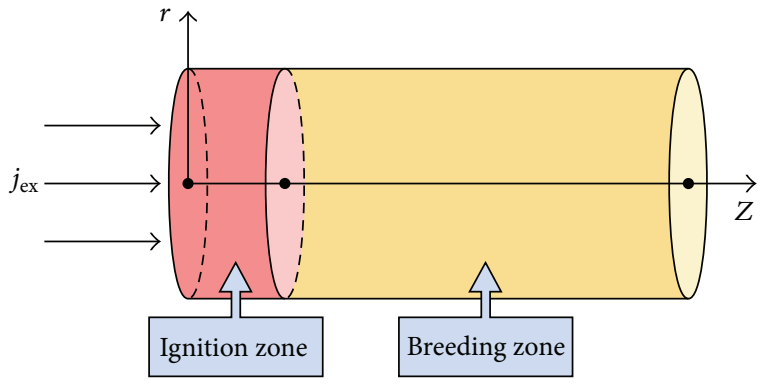

FIGURE 4: Schematic sketch of the two-zone cylindrical TWR.

And finally, there is one more important conclusion. From the analysis of (41) and (42) it is clear that the initial parameter $k_{0}$ for burning zone (Figure 4 ) is predefined solely by the nuclear burning wave burnup conditions, that is, by the parameters of an external neutron source and burnup area composition (Figure 4). In other words, it means that by tuning the corresponding burnup conditions for a given nuclear fuel composition, we can set the certain value of the nuclear burning front width. Moreover, by selecting the corresponding equilibrium $\widetilde{n}_{\text {fis }}$ and critical $n_{\text {crit }}$ concentrations of the active nuclear fuel component, we can define the required value of the nuclear burning wave speed $u$. Hence it is an obvious way for us to control the corresponding neutron fluence $\tau_{\varphi}$ accumulation time in the cladding material of the TWR fuel rod.

Hence we can make an important conclusion that the realization of the TWR with inherent safety requires the knowledge about the physics of nuclear burning wave burnup and the interrelation between the speed of nuclear burning wave and the fuel composition. As shown in [5], the properties of the fuel are completely defined by the equilibrium $\tilde{n}_{\text {fis }}$ and critical $n_{\text {crit }}$ (see (3)) concentrations of the active nuclear fuel component. We examine this in more detail below.

\section{Control Parameter and Condition of Existence of Stationary Wave of Nuclear Burning}

The above stated raises a natural question: "What does the nuclear burning wave speed in uranium-plutonium (1) and thorium-uranium (2) cycles mainly depend on?" The answer is rather simple and obvious. The nuclear burning wave speed in both cycles (far away from the burnup source) is completely characterized by its equilibrium $\widetilde{n}_{\text {fis }}$ and critical $n_{\text {crit }}$ concentrations of the active fuel component.

First of all, this is determined by a significant fact that the equilibrium $\tilde{n}_{\text {fis }}$ and critical $n_{\text {crit }}$ concentrations of the active fuel component completely identify the neutron-multiplying properties of the fuel environment. They are the conjugate pair of the integral parameters, which, due to their physical content, fully and adequately characterize all the physics of the nuclear transformations predefined by the initial fuel composition. This is also easy to see from a simple analysis of the kinetics equations solutions for the neutrons and nuclei, used in different models [5-23]. It means that regardless of the nuclear cycle type and initial fuel composition, the nuclear burning wave speed is defined by the equilibrium $\widetilde{n}_{\text {fis }}$ and critical $n_{\text {crit }}$ concentrations of the active fuel component through the so-called para-parameter a (see (3)). Consequently, as the numerical simulation results show (Figure 5 [5]), it follows the Wigner statistics.

It is important to note that each of these concentrations varies during the nuclear burning, but their ratio

$$
a=\frac{\pi}{2} \sqrt{\frac{n_{\text {crit }}}{\tilde{n}_{\text {fis }}-n_{\text {crit }}}}
$$

is a characteristic constant value for the given nuclear burning process [5]. In addition to that this para-parameter also determines (and it is extremely important!) the conditions for the nuclear burning wave existence (3), the neutron nuclear 


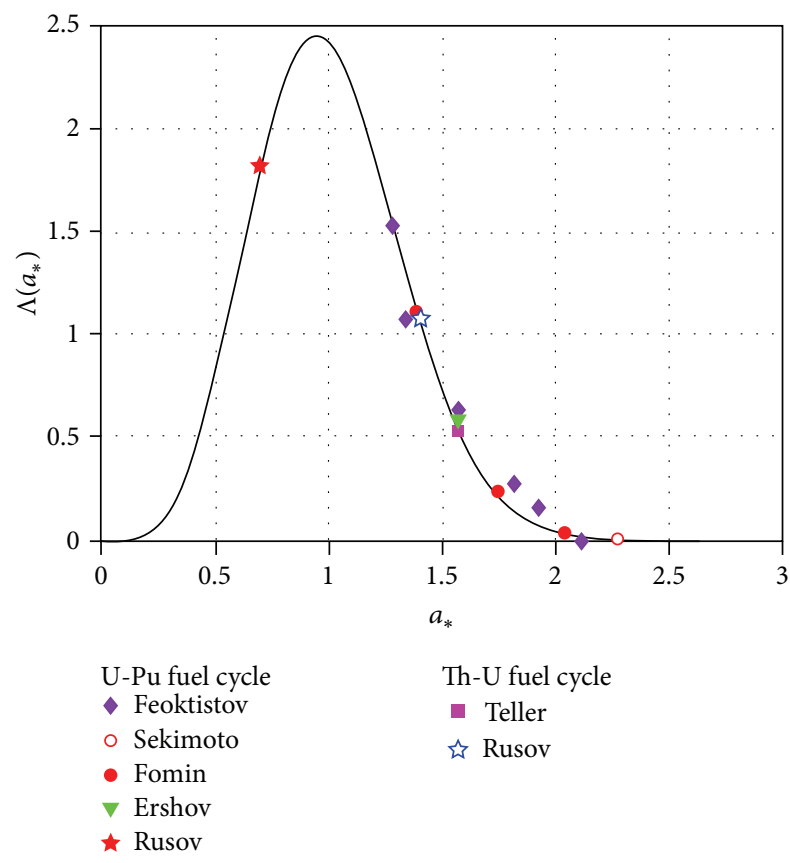

FIgURE 5: The theoretical (solid line) and calculated (points) dependence of $\Lambda\left(a_{*}\right)=u \tau_{\beta} / 2 L$ on the parameter $a$ [5].

burning wave speed (see (3)), and the dimensionless width (54) of the supercritical area in the burning wave of the active nuclear fuel component.

Therefore, when Pavlovich et al. [14-17] state that they control the values of the parameters with purposeful variation of the initial reactor composition, it actually means that, changing the effective concentration of the absorbent, they purposefully and definitely change (by definition (see [3, 5])) the equilibrium and critical concentrations of the active component, that is, the para-parameter (54) of the nuclear TWR-burning process.

It is necessary to note that the adequate understanding of the control parameter determination problem is not a simple or even a scholastic task, but is extremely important for the effective solution of another problem (the major one, in fact!), related to investigation of the nuclear burning wave stability conditions. In our opinion, the specified condition for the stationary nuclear burning wave existence (3) from [5] as well as the one obtained by Pavlovich group reveals the path to a sensible application of the so-called direct Lyapunov method [33,34] (the base theory for the movement stability) and thus the path to a reliable justification of the Lyapunov functional minimum existence (if it does exist) [33-37]. Some variants of a possible solution stability loss due to anomalous evolution of the nuclear fuel temperature are considered in Section 5 .

At the same time one may conclude that the "differential" [5] and "integral" [14-16] conditions for the stationary nuclear burning wave existence provide a complete description of the wave reactor physics and can become a basis for the future engineering calculations of a contemporary TWR project with the optimal or preset wave properties.

\section{On the Dependence of the Damaging Dose on Neutron Fluence, Phase Velocity, and Dispersion of the Solitary Burnup Waves}

As follows from the expression for the soliton-like solution (46), it is defined by three parameters, the maximal neutron flux $\psi_{m}$, the phase $\alpha$, and the speed $u$ of nuclear burning wave. And even if we can control them, it is still unclear which condition determines the optimal values of these parameters. Let us try to answer this question shortly.

It is known that high cost effectiveness and competitiveness of the fast reactors, including TWR, may be achieved only in case of a high nuclear fuel burnup (since the maximum burnup of the FN-600 reactor is currently 10\% [38], the burnup degree of $\sim 20 \%$ for the TWR may be considered more than acceptable). As the experience of the fast reactors operation shows, the main hindrance in achieving the high nuclear fuel burnup is the insufficient radiation resistance of the fuel rod shells. Therefore the main task of the radiation material science (along with the study of the physics behind the process) is to create a material (or select among the existing materials), which would keep the required level of performance characteristics being exposed to the neutron irradiation. One of the most significant phenomena leading to a premature fuel rods destruction is the void swelling of the shell material [39-42]. Moreover, the absence of the swelling saturation at an acceptable level and its acceleration with the damaging dose increase lead to a significant swelling (volume change up to $30 \%$ and more) and subsequently to a significant increase of the active zone elements size. The consequences of such effect are amplified by the fact that the high sensitivity of the swelling to temperature and irradiation damaging dose leads to distortions of the active zone components form because of the temperature and irradiation gradients. And finally, one more aggravating consequence of the high swelling is almost complete embrittlement of the construction materials at certain level of swelling (it is known that the fuel rod shell diameter increase due to swelling is accompanied by an anomalously high corrosion damage of the shell by the fuel [38]). Consequently, in order to estimate the possible amount of swelling, a damaging dose (measured in $\mathrm{dpa}$ ) initiated by the fast neutrons, for example, in the fuel rod shells, must be calculated (Figure 6).

Usually in order to evaluate the displacements per atom (DPA) created by the spallation residues, the so-called modified NRT method is applied $[44,45]$, which takes into account the known Lindhard correction [46]. Within such modified NRT method, the total number of displacements produced by the residues created by spallation reactions in the energetic window can be calculated as the addition of the displacements produced by each of these residues $(Z, A)$ that in its turn leads to the following expression for the displacements per atom:

$$
n_{\mathrm{dpa}}=t \cdot \sum_{i}^{N}\left\langle\sigma_{\mathrm{dpa}}^{i}\right\rangle \int_{E_{i}-1}^{E_{i}} \Phi\left(E_{i}\right) d E_{i}=\sum_{i=1}^{N}\left\langle\sigma_{\mathrm{dpa}}^{i}\right\rangle \varphi_{i} t
$$

where

$$
\left\langle\sigma_{\text {dpa }}^{i}\right\rangle\left(E_{i}\right)=\left\langle\sigma_{d}\left(E_{i}, Z, A\right)\right\rangle \cdot d\left(E_{d}(Z, A)\right),
$$




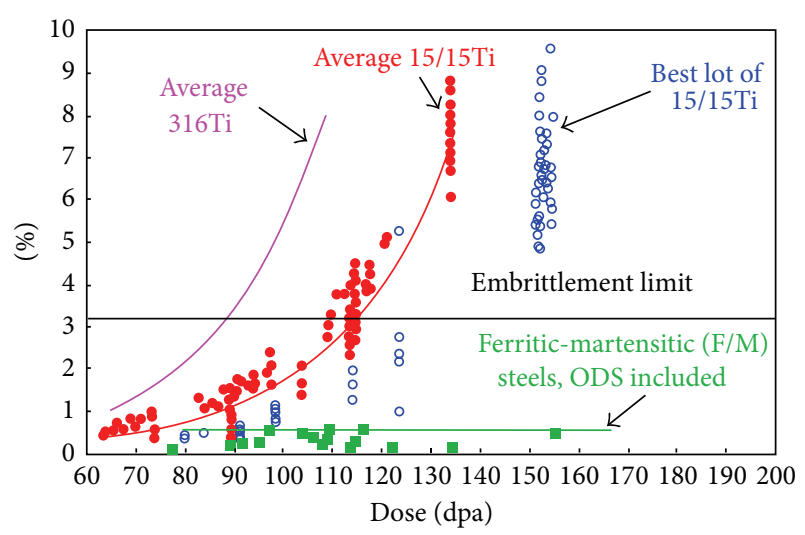

FIgURE 6: Swelling of austenitic Phenix cladding compared with ferritic-martensitic materials, ODS included, adopted from [43].

while $\sigma_{d}\left(E_{i}, Z, A\right)$ is the displacement cross section of recoil atom $(Z, A)$, produced at incident particle energy $E_{i}, \Phi(E)$ is the energy-dependent flux of incident particles during time $t$ and $d\left(E_{d}(Z, A)\right)$ is the number of displacements created at threshold displacement energy $E_{d}$ of recoil atom $(Z, A)$ or its so-called damage function.

Actually, all the recoil energy of the residue $E_{r}$ is not going to be useful to produce displacements because a part of it lost inelastic scattering with electrons in the medium. An estimation of the damage energy of the residue can be calculated using the Lindhard factor $\xi$ [46]:

$$
E_{\mathrm{dam}}=E_{r} \xi
$$

The number of displacements created by a residue $(Z, A)$ is calculated using this damage energy (57) and the NRT formula:

$$
d\left(E_{d}(Z, A)\right)=\eta \frac{E_{\mathrm{dam}}}{2\left\langle E_{d}\right\rangle},
$$

where $\left\langle E_{d}\right\rangle$ is the average threshold displacement energy of an atom to its lattice site, $\eta=0.8[44,45]$.

Consequently the condition of the maximal damaging dose for the cladding materials of the fast neutron reactors, taking into account the metrological data of IAEA [47] (see Figure 7) and contemporary estimates by Pukari and Wallenius (see yellow inset at Figure 6), takes the form:

$$
n_{\mathrm{dpa}} \simeq\left\langle\sigma_{\mathrm{dpa}}\right\rangle \cdot \varphi \cdot \frac{2 \Delta_{1 / 2}}{u} \leqslant 200[\mathrm{dpa}] .
$$

In this case the selection strategy for the required wave parameters and allowed values of the neutron fluence for the future TWR project must take into account the condition of the maximal damaging dose (58) for cladding materials in fast neutron reactors and, therefore, must comply with the following dpa-relation:

$$
\left\langle\sigma_{\mathrm{dpa}}\right\rangle \cdot \varphi \cdot \frac{\Delta_{1 / 2}}{u} \leqslant 100[\mathrm{dpa}] .
$$

The question here is whether or not the parameters of the wave and neutron fluence which can provide the burnup

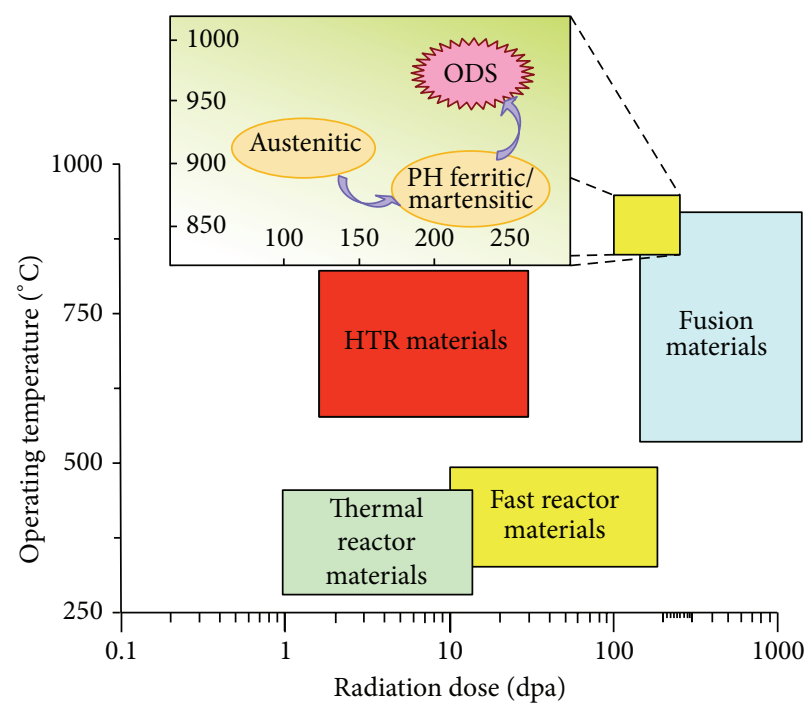

FIgURE 7: Operating condition for core structural materials in different power reactors [47]. The upper yellow inset represents the data of Pukari and Wallenius [43].

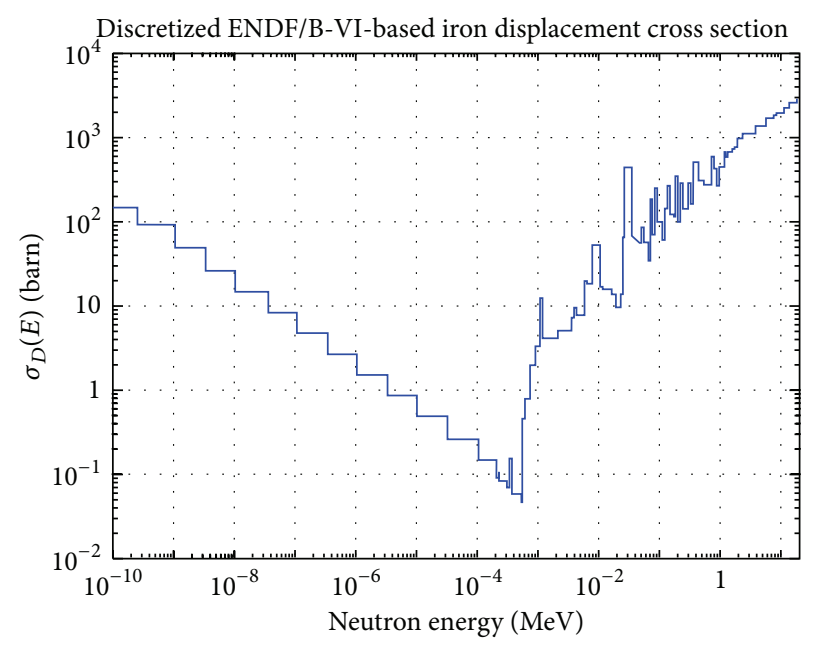

FIGURE 8: Discretized displacement cross section for stainless steel based on the Lindhard model and ENDF/B scattering cross section, adopted from [48].

level of the active nuclear fuel component in TWR-type fast reactor of at least $20 \%$ are possible. Since we are interested in the cladding materials resistible to the fast neutron damaging dose, we will assume that the displacement cross section for the stainless steel, according to Mascitti and Madariaga [48] for neutrons with average energy $2 \mathrm{MeV}$, equals $\left\langle\sigma_{\mathrm{dpa}}\right\rangle \approx$ 1000 dpa (Figure 8).

The analysis of the nuclear burning wave parameters, in some authors' models of TWR presented in Table 1, shows that in the case of $\mathrm{U}-\mathrm{Pu}$ cycle none of the considered models satisfy the dpa-parameter, while two Th-U cycle models by Teller et al. [4] and Seifritz [6] groups correspond well to the major requirements to wave reactors. 
TABLE 1: Results of the numerical experiments of the wave mode parameters based on U-Pu and Th-U cycles.

\begin{tabular}{|c|c|c|c|c|c|c|c|c|}
\hline & $\Delta_{1 / 2}[\mathrm{~cm}]$ & $u[\mathrm{~cm} /$ day $]$ & $\varphi\left[\mathrm{cm}^{-2} \mathrm{~s}^{-1}\right]$ & $\psi\left[\mathrm{cm}^{-2}\right]$ & $\left\langle\sigma_{\text {dpa }}\right\rangle[$ barn] & $n_{\mathrm{dpa}} / 200$ & Fuel burn-up & Solution \\
\hline \multicolumn{9}{|c|}{ U-Pu cycle } \\
\hline Sekimoto and Udagawa [12] & 90 & 0.008 & $3.25 \cdot 10^{15}$ & $3.2 \cdot 10^{23}$ & 1000 & 3.2 & $\sim 43 \%$ & No \\
\hline Rusov et al. [5] & 200 & 2.77 & $10^{18}$ & $6.2 \cdot 10^{24}$ & 1000 & 62 & $\sim 60 \%$ & No \\
\hline Pavlovich et al. [16] & - & 0.003 & - & $1.7 \cdot 10^{24}$ & 1000 & 17 & $\sim 30 \%$ & No \\
\hline Fomin et al. [49] & 100 & 0.07 & $2 \cdot 10^{16}$ & $2.5 \cdot 10^{24}$ & 1000 & 25 & $\sim 30 \%$ & No \\
\hline Fomin et al. [50] & 125 & 1.7 & $5 \cdot 10^{17}$ & $3.2 \cdot 10^{24}$ & 1000 & 32 & $\sim 40 \%$ & No \\
\hline Chen et al. [18] & 216 & 0.046 & $3 \cdot 10^{15}$ & $1.2 \cdot 10^{24}$ & 1000 & 12 & $\sim 30 \%$ & No \\
\hline Weaver et al. [23] & - & - & - & - & - & 1.75 & $\sim 20 \%$ & No \\
\hline \multicolumn{9}{|c|}{ Th-U cycle } \\
\hline Teller et al. [4] & 70 & 0.14 & $\sim 2 \cdot 10^{15}$ & $8.6 \cdot 10^{22}$ & 1000 & 0.96 & $\sim 50 \%$ & Yes \\
\hline Seifritz [6] & 100 & 0.096 & $10^{15}$ & $9.0 \cdot 10^{22}$ & 1000 & 0.90 & $\sim 30 \%$ & Yes \\
\hline Melnik et al. [51] & 100 & 0.0055 & $0.5 \cdot 10^{16}$ & $7.9 \cdot 10^{24}$ & 1000 & $\sim 80$ & $\sim 50 \%$ & No \\
\hline \multicolumn{9}{|c|}{ U-Pu (+ moderator) } \\
\hline Example & 100 & 0.234 & $2.5 \cdot 10^{15}$ & $9.2 \cdot 10^{23}$ & 100 & 0.92 & $\sim 20 \%$ & Yes \\
\hline Ideal TWR & - & - & - & $10^{24}$ & 100 & 1.0 & $\geqslant 20 \%$ & Yes \\
\hline
\end{tabular}

TABLE 2: Moderating and absorbing properties of some substances, moderator layer width estimate for moderating neutron from $E_{\text {fuel }}=$ $1.0 \mathrm{MeV}$ to $E_{\bmod }=0.1 \mathrm{MeV}$.

\begin{tabular}{|c|c|c|c|c|c|c|}
\hline Moderator & Mass number, $A$ & $\begin{array}{l}\text { Mean logarithmic } \\
\text { energy, } \xi\end{array}$ & Density, $\rho, \mathrm{g} / \mathrm{cm}^{3}$ & $\begin{array}{c}\text { Impacts number } \\
\text { required for } \\
\text { moderating, } n\end{array}$ & $\begin{array}{l}\text { Neutron mean free } \\
\text { path, } \lambda\end{array}$ & $\begin{array}{l}\text { Moderator layer } \\
\text { width, } R_{\text {mod }}, \mathrm{cm}\end{array}$ \\
\hline $\mathrm{Be}$ & 9 & 0.21 & 1.85 & 11 & 1.39 & 15.3 \\
\hline $\mathrm{C}$ & 12 & 0.158 & 1.60 & 15 & 3.56 & 53.4 \\
\hline $\mathrm{H}_{2} \mathrm{O}$ & 18 & 0.924 & 1.0 & 2.5 & 16.7 & 41.6 \\
\hline $\mathrm{H}_{2} \mathrm{O}+\mathrm{B}$ & & & & 2.5 & 10.0 & 25.0 \\
\hline $\mathrm{He}$ & 4 & 0.425 & 0.18 & 5.41 & 11.2 & 60.7 \\
\hline
\end{tabular}

On the other hand, these authors apparently did not take the problem of dpa-parameter in cladding materials into account, since they were mainly interested in the fact of the wave mode of nuclear burning existence in $\mathrm{U}-\mathrm{Pu}$ and Th-U cycles at the time.

However, as the analysis of Table 1 shows, the procedure accounting for the dpa-parameter is not problematic, but it leads to unsatisfactory results relative to the burnout of the main fissile material. It follows that when $\left\langle\sigma_{\mathrm{dpa}}\right\rangle \approx 1000 \mathrm{dpa}$, the dpa-condition considered above for the maximum possible damaging dose for cladding materials of the fast neutron reactors

$$
\psi_{1000}=\frac{\Delta_{1 / 2}}{u} \varphi \simeq 10^{23}\left[\mathrm{~cm}^{-2}\right]
$$

is not met by any example in the table. Here $\psi_{1000}$ is the neutron fluence in case $\left\langle\sigma_{\mathrm{dpa}}\right\rangle \approx 1000 \mathrm{dpa}, \varphi$ is the neutron flux, and $\Delta_{1 / 2}$ and $u$ are the width and speed of the solitonlike nuclear burning wave.

So on the one hand, the neutron fluence must be increased by an order of magnitude to increase the burnup level significantly, and, on the other hand, the maximum damaging dose for the cladding materials must also be reduced by an order of magnitude. Such a controversial condition may be fulfilled considering that the reduction of the fuel rod shell radioactive damage for a given amount may be achieved by reducing the neutron flux density and energy (see Figure 8 ). The latter is achieved by placing a specially selected substance between fissile medium and fuel rod shell, which has the suitable characteristics of neutron moderator and absorbent.

At the same time it is known from the reactor neutron physics $[29,52]$ that the moderator layer width estimate $R_{\bmod }$ is

$$
R_{\bmod } \simeq \frac{1}{\Sigma_{S}+\Sigma_{a}} \cdot \frac{1}{\xi} \ln \frac{E_{\text {fuel }}}{E_{\text {mod }}}
$$

where $\Sigma_{S} \approx\left\langle\sigma_{S}\right\rangle N_{\bmod }$ and $\Sigma_{a} \approx\left\langle\sigma_{a}\right\rangle N_{\bmod }$ are the macroscopic neutron scattering and absorption cross sections, respectively, $\left\langle\sigma_{S}\right\rangle$ and $\left\langle\sigma_{a}\right\rangle$ are the microscopic neutron scattering and absorption cross sections, respectively, averaged by energy interval of the moderating neutrons from $E_{\text {fuel }}=2 \mathrm{MeV}$ to $E_{\text {mod }}=0.1 \mathrm{MeV}, N_{\bmod }$ is the moderator nuclei density, and $\xi=1+(A+1)^{2} \ln [(A-1) / A+1] / 2 A$ is the neutron energy decrement of its moderation in the moderator-absorbent medium with atomic number $A$.

It is clearly seen that the process of neutron moderation from 2.0 MeV to $0.1 \mathrm{MeV}$ energy in moderator-absorbent of a given width (see Table 2) creates a new but satisfactory level of maximum possible damaging dose for the cladding materials, 
corresponding to $\left\langle\sigma_{\mathrm{dpa}}\right\rangle \approx 100 \mathrm{dpa}$ (Figure 8 ). Therefore if we are satisfied with the main fissile material burnout level around $\sim 20 \%$, then, analyzing Tables 1 and 2 , the conditions accounting for the dpa-parameter problem and contemporary level of the radioactive material science will have the following form:

$$
\psi_{100}=\frac{\Delta_{1 / 2}}{u} \varphi \simeq 10^{24}\left[\mathrm{~cm}^{-2}\right],
$$

where $\psi_{100}$ is the neutron fluence (with $0.1 \mathrm{MeV}$ energy) on cladding materials surface in case $\left\langle\sigma_{\text {dpa }}\right\rangle \approx 100 \mathrm{dpa}$ (see Figure 8 and "ideal" case in Table 1).

And finally one can make the following intermediate conclusion. As shown above in Section 3, the algorithm for determining the parameters (61) is mainly defined by paraparameter that plays a role of a "response function" to all the physics of nuclear transformations, predefined by initial fuel composition. It is also very important that this parameter unequivocally determines the conditions of the nuclear burning wave existence (3), the neutron nuclear burning wave speed (see (3)), and the dimensionless width (54) of the supercritical area in the wave of the active component burning.

Based on the para-parameter ideology [5] and Pavlovych group results [14-16], we managed to pick up a mode for the nuclear burning wave in $\mathrm{U}-\mathrm{Pu}$ cycle, having the parameters shown in Table 1 satisfyied (61). The latter means that the problem of dpa-parameter in cladding materials in the TWRproject is currently not an insurmountable technical problem and can be successfully solved.

In our opinion, the major problem of TWR is the socalled temperature blow-up modes that take place due to coolant loss as observed during Fukushima nuclear accident. Therefore below we will consider the possible causes of the TWR inherent safety breach due to temperature blow-up mode.

\section{Possible Causes of the TWR Inherent Safety Failure: Fukushima Plutonium Effect and the Temperature Blowup Mode}

It is known that with loss of coolant at three nuclear reactors during the Fukushima nuclear accident its nuclear fuel melted. It means that the temperature in the active zone reached the melting point of uranium-oxide fuel at some moments (note that the third block partially used MOX-fuel enriched with plutonium), that is, $\sim 3000 \mathrm{~K}$.

Surprisingly enough, in scientific literature today there are absolutely no either experimental or even theoretically calculated data on behavior of the ${ }^{238} \mathrm{U}$ and ${ }^{239} \mathrm{Pu}$ capture cross sections depending on temperature at least in 1000$3000 \mathrm{~K}$ range. At the same time there are serious reasons to suppose that the cross section values of the specified elements increase with temperature. We may at least point out qualitative estimates by Ukraintsev [53], Obninsk Institute of Atomic Energetics (Russia), that confirm the possibility of the cross sections' growth for ${ }^{239} \mathrm{Pu}$ in $300-1500 \mathrm{~K}$ range.

Obviously, such anomalous temperature dependency of capture and fission cross sections of ${ }^{238} \mathrm{U}$ and ${ }^{239} \mathrm{Pu}$ may change the neutron and thermal kinetics of a nuclear reactor drastically, including the perspective fast uranium-plutonium new generation reactors (reactors of Feoktistov (1) and Teller (2) type), which we classify as fast TWR reactors. Hence it is very important to know the anomalous temperature behavior of ${ }^{238} \mathrm{U}$ and ${ }^{239} \mathrm{Pu}$ capture and fission cross sections as well as their influence on the heat transfer kinetics, because it may turn into a reason of the positive feedback (positive feedback is a type of feedback when a change in the output signal leads to such a change in the input signal, which leads to even greater deviation of the output signal from its original value. In other words, PF leads to the instability and appearance of qualitatively new (often self-oscillation) systems) (PF) with the neutron kinetics leading to an undesirable solution stability loss (the nuclear burning wave) and consequently to a trivial reactor runaway with a subsequent nontrivial catastrophe.

A special case of the PF is a nonlinear PF, which leads to the system evolution in the so-called blowup mode [5459] or, in other words, in such a dynamic mode when one or several modeled values (e.g., temperature and neutron flux) grow to infinity at a finite time. In reality, instead of the infinite values, a phase transition is observed in this case, which can become a first stage or a precursor of the future technogenic disaster.

Investigation of the temperature dependency of ${ }^{238} \mathrm{U}$ and ${ }^{239} \mathrm{Pu}$ capture and fission cross sections in $300-3000 \mathrm{~K}$ range and the corresponding kinetics of heat transfer and its influence on neutron kinetics in TWR is the main goal of the section.

Heat transfer equation for uranium-plutonium fissile medium is as follows:

$$
\begin{aligned}
\rho(\vec{r}, T, t) \cdot c(\vec{r}, T, t) \cdot \frac{\partial T(\vec{r}, t)}{\partial t} \\
=\aleph(\vec{r}, T, t) \cdot \Delta T(\vec{r}, t)+\nabla \aleph(\vec{r}, T, t) \cdot \nabla T(r, t) \\
\quad+q_{T}^{f}(\vec{r}, T, t),
\end{aligned}
$$

where the effective substance density is

$$
\rho(\vec{r}, T, t)=\sum_{i} N_{i}(\vec{r}, T, t) \cdot \rho_{i}
$$

$\rho_{i}$ are tabulated values and $N_{i}(\vec{r}, T, t)$ are the components concentrations in the medium, while the effective specific heat capacity (accounting for the medium components heat capacity values $c_{i}$ ) and fissile material heat conductivity coefficient (accounting for the medium components heat conductivity coefficients $\aleph_{i}(T)$ ), respectively, are

$$
\begin{aligned}
c(\vec{r}, T, t) & =\sum_{i} c_{i}(T) N_{i}(\vec{r}, T, t), \\
\aleph(\vec{r}, T, t) & =\sum_{i} \aleph_{i}(T) N_{i}(\vec{r}, T, t) .
\end{aligned}
$$

Here $q_{T}^{f}(\vec{r}, T, t)$ is the heat source density generated by the nuclear fissions $N_{i}$ of fissile metal components that vary in time. 
Theoretical temperature dependency of heat capacity $c(T)$ for metals is known, at low temperatures $c(t) \sim T^{3}$ and at high temperatures $c(T) \rightarrow$ const, and the constant value (const $\approx$ $6 \mathrm{Cal} /(\mathrm{mol} \cdot \mathrm{deg}))$ is determined by Dulong-Petit law. At the same time it is known that the thermal expansion coefficient is small for metals; therefore, the specific heat capacity at constant volume $c_{v}$ is almost equal to the specific heat capacity at constant pressure $c_{p}$. On the other hand, the theoretical dependency of heat conductivity $\aleph_{i}(T)$ at high temperature of "fissile" metals is not known, while it is experimentally determined that the heat conductivity coefficient $\aleph(T)$ of fissile medium is a nonlinear function of temperature (e.g., see [60], where the heat conductivity coefficient is given for $\alpha$-uranium 238 and for metallic plutonium 239, and also [61]).

While solving the heat conduction equations we used the following initial and boundary conditions:

$$
T(r, t=0)=300 \mathrm{~K}, \quad j_{n}=\aleph\left[T(r \in \mathfrak{R}, t)-T_{0}\right],
$$

where $j_{n}$ is the normal (to the fissile medium boundary) heat flux density component, $\aleph(T)$ is the thermal conductivity coefficient, $\mathfrak{R}$ is the fissile medium boundary, and $T_{0}$ is the temperature of the medium adjacent to the active zone.

Obviously, if the cross sections of some fissile nuclides increase, then, due to the nuclei fission reaction exothermicity, the direct consequence of the significantly nonlinear kinetics of the parental and child nuclides in the nuclear reactor is an autocatalyst increase of generated heat, similar to autocatalyst processes of the exothermic chemical reactions. In this case the heat flux density $q_{T}^{f}(\vec{r}, \Phi, T, t)$ that characterizes the generated heat amount will be

$$
\begin{aligned}
q_{T}^{f} & (\vec{r}, \Phi, T, t) \\
& =\Phi(\vec{r}, T, t) \sum_{i} Q_{i}^{f} \bar{\sigma}_{f}^{i}(\vec{r}, T, t) N_{i}(\vec{r}, T, t)\left[\mathrm{W} / \mathrm{cm}^{3}\right],
\end{aligned}
$$

where

$$
\Phi(\vec{r}, T, t)=\int_{0}^{E_{n}^{\max }} \Phi(\vec{r}, E, T, t) d E
$$

is the full neutron flux density, $\Phi(\vec{r}, E, T, t)$ is the neutron flux density with energy $E, Q_{i}^{f}$ is the mean generated heat emitted due to fission of one nucleus of the $i$ th nuclide,

$$
\bar{\sigma}_{f}^{i}(\vec{r}, T, t)=\int_{0}^{E_{n}^{\max }} \sigma_{f}^{i}(E, T) \rho(\vec{r}, E, T, t) d E
$$

is the fission cross section of the $i$ th nuclide averaged over the neutron spectrum,

$$
\rho(\vec{r}, E, T, t)=\frac{\Phi(\vec{r}, E, T, t)}{\Phi(\vec{r}, T, t)}
$$

is the neutron energy distribution probability density function, $\sigma_{f}^{i}(E, T)$ is the microscopic fission cross section of the $i$ th nuclide that, as known, depends on the neutron energy and fissile medium temperature (Doppler effect [29]), and $N_{i}(\vec{r}, T, t)$ is the density of the $i$ th nuclide nuclei.

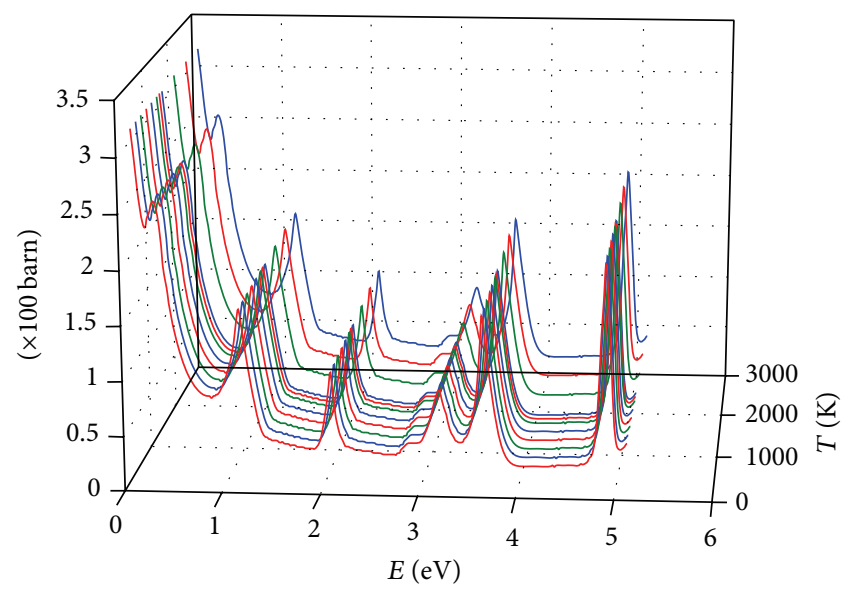

FIGURE 9: Calculated dependency of radioactive neutron capture cross section on the energy for ${ }_{92}^{235} \mathrm{U}$ at different temperatures within $300 \mathrm{~K}$ to $3000 \mathrm{~K}$.

As follows from (69), in order to build the thermal source density function it is necessary to derive the theoretical dependency of the cross sections $\bar{\sigma}_{f}^{i}(\vec{r}, T, t)$, averaged over the neutron spectrum, on the reactor fuel temperature. As is known, the influence of the nuclei thermal motion on the medium comes to a broadening and height reduction of the resonances. By optical analogy, this phenomenon is referred to as Doppler effect [29]. Since the resonance levels are observed only for heavy nuclei in the low energy area, then Doppler effect is notable only during the interaction of neutrons with such nuclei. And the higher the environment temperature is the stronger the effect is.

Therefore a program was developed using Microsoft Fortran Power Station 4.0 (MFPS 4.0) that allows at the first stage the cross sections of the resonance neutron reactions to be calculated depending on neutron energy taking into account the Doppler effect. The cross sections dependency on neutron energy for reactor nuclides from ENDF/B-VII database [62], corresponding to $300 \mathrm{~K}$ environment temperature, was taken as the input data for the calculations. For example, the results of radioactive neutron capture cross sections dependency on neutron energy for ${ }^{235} \mathrm{U}$ are given in Figure 9 for different temperatures of the fissile medium in $300 \mathrm{~K}-3000 \mathrm{~K}$ temperature range. Using this program, the dependency of scattering fission and radioactive neutron capture cross sections for the major reactor fuel nuclides ${ }_{92}^{235} \mathrm{U},{ }_{92}^{238} \mathrm{U},{ }_{92}^{239} \mathrm{U}$, and ${ }_{94}^{239} \mathrm{Pu}$ for different temperatures in range $300 \mathrm{~K}$ to $3000 \mathrm{~K}$ was obtained.

At the second stage a program was developed to obtain the calculated dependency of the cross sections $\bar{\sigma}_{f}^{i}(\vec{r}, T, t)$ averaged over the neutron spectrum for main reactor nuclides and for main neutron reactions for the specified temperatures. The averaging of the neutron cross sections for the Maxwell distribution was performed using the following expression:

$$
\left\langle\sigma\left(E_{\lim }, T\right)\right\rangle=\frac{\int_{0}^{E_{\lim }} E^{1 / 2} e^{-E / k T} \sigma(E, T) d E}{\int_{0}^{E_{\lim }} E^{1 / 2} e^{-E / k T} d E},
$$


where $E_{\text {lim }}$ is the upper limit of the neutrons thermalization, while for the procedure of neutron cross sections averaging over the Fermi spectrum the following expression was used:

$$
\left\langle\sigma\left(E_{\lim }, T\right)\right\rangle=\frac{\int_{E_{\lim }}^{\infty} \sigma(E, T) E^{-1} d E}{\int_{E_{\lim }}^{\infty} E^{-1} d E} .
$$

During further calculations in our programs we used the results obtained at the first stage, that is, the dependency of reaction cross sections on neutron energy and environment temperature (Doppler effect). The neutron spectrum was specified in a combined way by Maxwell spectrum $\Phi_{M}\left(E_{n}\right)$ below the limit of thermalization $E_{\text {lim }}$, by Fermi spectrum $\Phi_{F}(E)$ for a moderating medium with absorption above $E_{\text {lim }}$ but below $E_{F}$ (upper limit for Fermi neutron energy spectrum), and by ${ }^{239} \mathrm{Pu}$ fission spectrum $[15,21]$ above $E_{F}$ but below the maximal neutron energy $E_{n}^{\max }$. Here the neutron gas temperature for Maxwell distribution was given by (75), described in [29]. According to this approach [29], the drawbacks of the standard slowing-down theory for thermalization area may be formally reduced if a variable $\xi(x)=\xi(1-2 / z)$ is introduced instead of the average logarithmic energy loss $\xi$, which is almost independent of the neutron energy (as known, the statement $\xi \approx 2 / A$ is true for the environment consisting of nuclei with $A>10$ ). Here $z=E_{n} / k T$, where $E_{n}$ is the neutron energy and $T$ is the environment temperature. Then the following expression may be used for the neutron gas temperature in Maxwell spectrum of thermal neutrons (a very interesting expression revealing hidden connection between the temperature of a neutron gas and the environment (fuel) temperature):

$$
T_{n}=T_{0}\left[1+\eta \cdot \frac{\Sigma_{a}\left(k T_{0}\right)}{\langle\xi\rangle \Sigma_{S}}\right]
$$

where $T_{0}$ is the fuel environment temperature, $\Sigma_{a}\left(k T_{0}\right)$ is an absorption cross section for energy $k T_{0}, \eta=1.8$ is the dimensionless constant, and $\langle\xi\rangle$ is averaged over the whole energy interval of Maxwell spectrum $\xi(z)$ at $k T=1 \mathrm{eV}$.

Fermi neutron spectrum for a moderating medium with absorption (we considered carbon as a moderator and ${ }^{238} \mathrm{U}$, ${ }^{239} \mathrm{U}$, and ${ }^{239} \mathrm{Pu}$ as the absorbers) was set in the form $[29,52]$ :

$$
\Phi_{\mathrm{Fermi}}\left(E, E_{F}\right)=\frac{S}{\langle\xi\rangle \Sigma_{t} E} \exp \left[-\int_{E_{\mathrm{lim}}}^{E_{f}} \frac{\Sigma_{a}\left(E^{\prime}\right) d E^{\prime}}{\langle\xi\rangle \Sigma_{t}\left(E^{\prime}\right) E^{\prime}}\right],
$$

where $S$ is the total volume neutron generation rate, $\langle\xi\rangle=$ $\sum_{i}\left(\xi_{i} \Sigma_{S}^{i}\right) / \Sigma_{S}, \xi_{i}$ is the average logarithmic decrement of energy loss, $\Sigma_{S}^{i}$ is the macroscopic scattering cross section of the $i$ th nuclide, $\Sigma_{t}=\sum_{i} \Sigma_{S}^{i}+\Sigma_{a}^{i}$ is the total macroscopic cross section of the fissile material, $\Sigma_{S}=\sum_{i} \Sigma_{S}^{i}$ is the total macroscopic scattering cross section of the fissile material, $\Sigma_{a}$ is the macroscopic absorption cross section, and $E_{F}$ is the upper neutron energy for Fermi spectrum.

The upper limit of neutron thermalization $E_{\text {lim }}$ in our calculation was considered a free parameter, setting the neutron fluxes of Maxwell and Fermi spectra at a common energy limit $E_{\lim }$ equal to

$$
\Phi_{\text {Maxwell }}\left(E_{\text {lim }}\right)=\Phi_{\text {Fermi }}\left(E_{\text {lim }}\right)
$$

The high energy neutron spectrum part $\left(E>E_{F}\right)$ was defined by the fission spectrum $[52,63,64]$ in our calculations. Therefore the following expression may be written for the total volume neutron generation rate $S$ in the Fermi spectrum (76):

$$
\begin{aligned}
& S(\vec{r}, T, t) \\
& =\int_{E_{F}}^{E_{n}^{\max }} \widetilde{P}(\vec{r}, E, T, t) \\
& \cdot\left[\sum_{i} v_{i}(E) \cdot \Phi(\vec{r}, E, T, t) \cdot \sigma_{f}^{i}(E, T) \cdot N_{i}(\vec{r}, T, t)\right] d E,
\end{aligned}
$$

where $E_{n}^{\max }$ is the maximum energy of the neutron fission spectrum (usually taken as $E_{n}^{\max } \approx 10 \mathrm{MeV}$ ), $E_{F}$ is the neutron energy, below which the moderating neutrons spectrum is described as Fermi spectrum (usually taken as $E_{F} \approx$ $0.2 \mathrm{MeV})$, and $\widetilde{P}(\vec{r}, E, T, t)$ is the probability of neutron not leaving the boundaries of the fissile medium which depends on the fissile material geometry and the conditions at its border (e.g., presence of a reflector).

The obtained calculation results show that the cross sections averaged over the spectrum may increase (Figure 10 for ${ }^{239} \mathrm{Pu}$ and Figure 12 for ${ }^{238} \mathrm{U}$ ) as well as decrease (Figure 11 for ${ }^{235} \mathrm{U}$ ) with fissile medium temperature. As follows from the obtained results, the arbitrariness in selection of the limit energy for joining the Maxwell and Fermi spectra does not significantly alter the character of these dependencies evolution.

This can be justified by the fact that ${ }^{239} \mathrm{Pu}$ resonance area starts from significantly lower energies than that of ${ }^{235} \mathrm{U}$, and with fuel temperature increase the neutron gas temperature also increases producing Maxwell's neutron distribution maximum shift to the higher neutron energies; that is, the neutron gas spectrum hardening, when more neutrons fit into resonance area of ${ }^{239} \mathrm{Pu}$, is the cause of the averaged cross sections growth.

This process is not as significant for ${ }^{235} \mathrm{U}$ because its resonance area is located at higher energies. As a result, the ${ }^{235} \mathrm{U}$ neutron gas spectrum hardening related to the fuel temperature increase (in the considered interval) does not result in a significant increase of a number of neutrons fitting into the resonance area. Therefore according to the known expressions of ${ }^{235} \mathrm{U}$ determining the neutron reactions cross sections behaviour depending on their energy $E_{n}$ for nonresonance areas, we observe dependency for the averaged cross sections $\sigma_{n b} \sim 1 / \sqrt{E_{n}}$.

The data on the averaged fission and capture cross sections of ${ }^{238} \mathrm{U}$ presented in Figure 12 show that the averaged fission cross section for ${ }^{238} \mathrm{U}$ is almost insensitive to the neutron spectrum hardening caused by the fuel temperature 


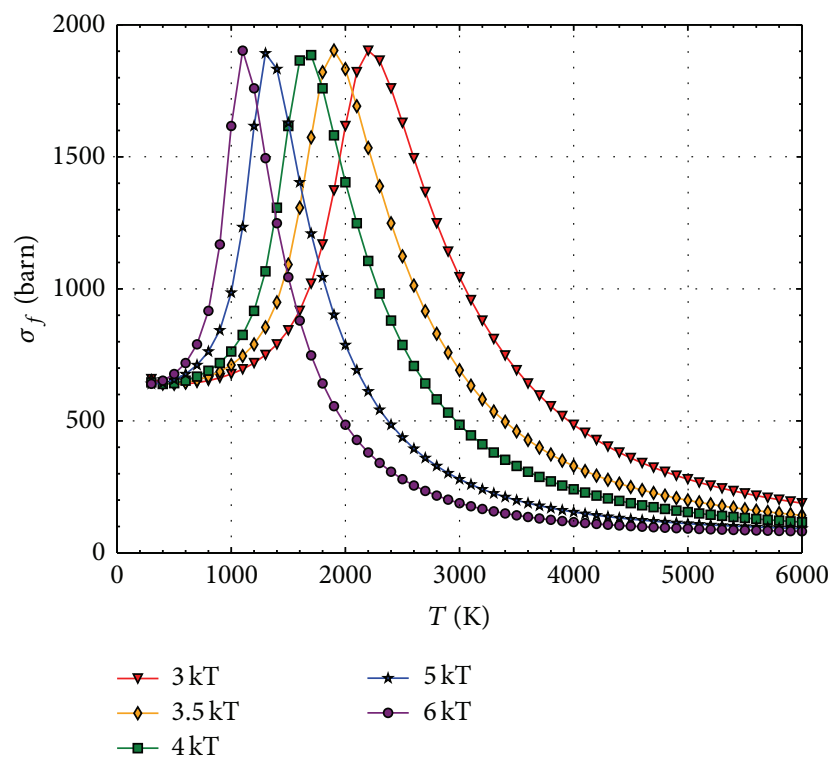

(a)

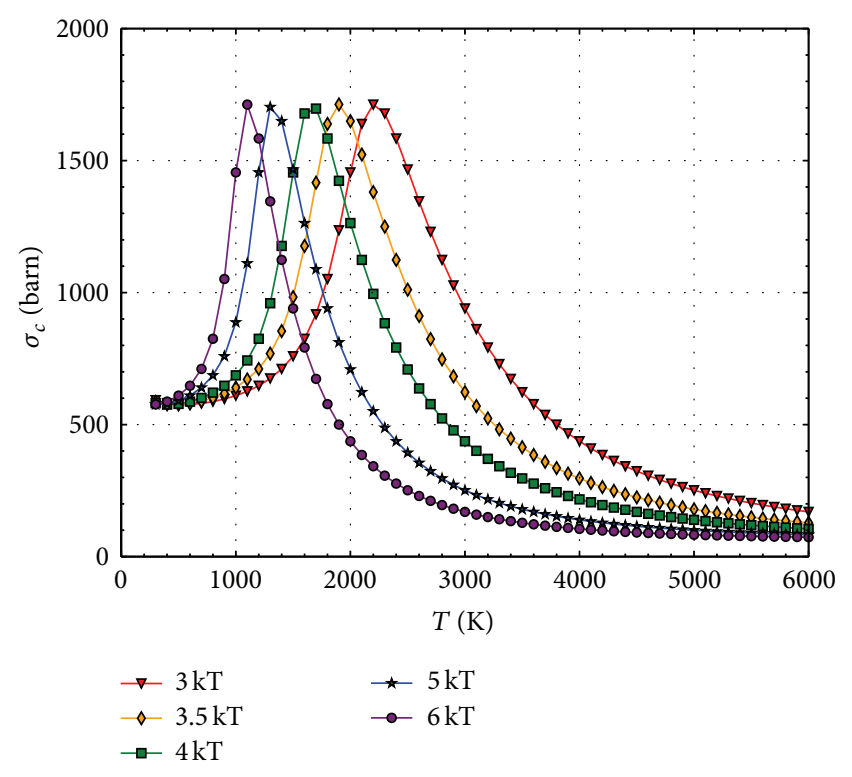

(b)

FIGURE 10: Temperature dependencies for the fission cross section (a) and radioactive capture cross section (b) for ${ }^{239} \mathrm{Pu}$, averaged over the Maxwell spectrum, on the Maxwell and Fermi spectra joining energy and $\eta=1.8$ (see (75)).

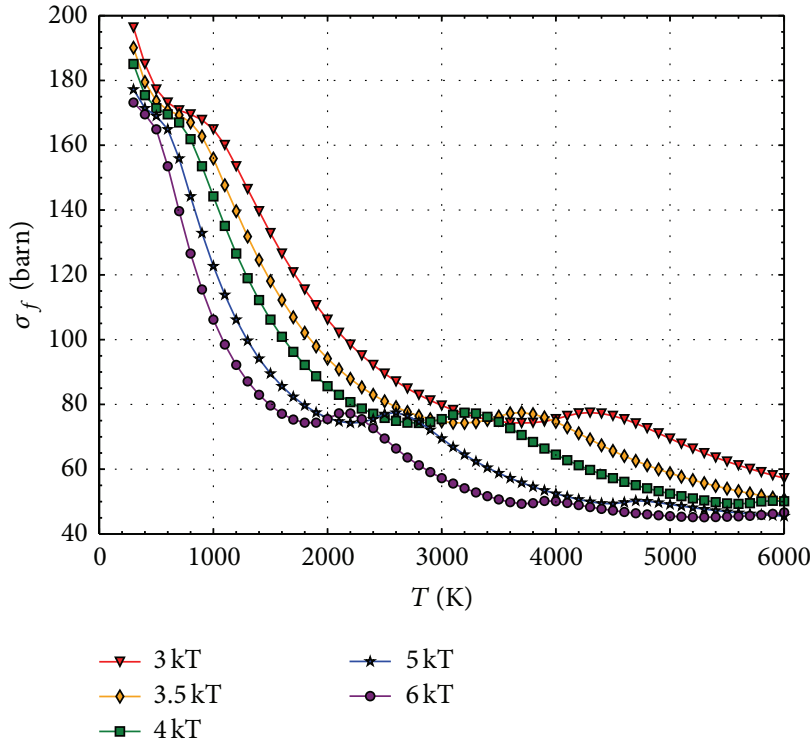

(a)

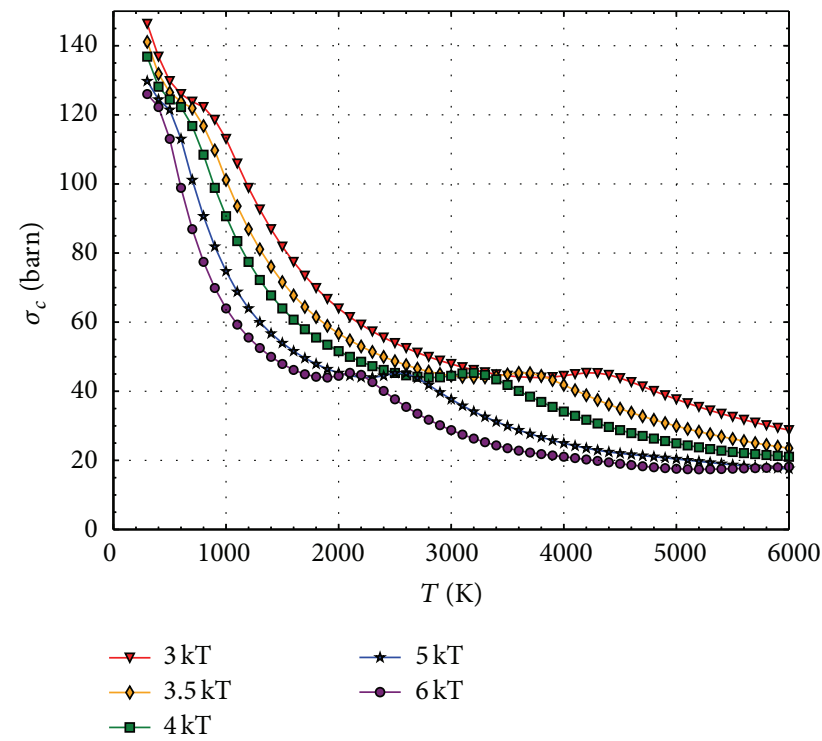

(b)

FIGURE 11: Temperature dependencies for the fission cross section (a) and radioactive capture cross section (b) for ${ }^{235} \mathrm{U}$, averaged over the Maxwell spectrum, on the Maxwell and Fermi spectra joining energy and $\eta=1.8$ (see (75)).

increase, due to a high fission threshold $\sim 1 \mathrm{MeV}$ (see Figure $12(\mathrm{a})$ ). At the same time they confirm the capture cross section dependence on temperature, since its resonance area is located as low as for ${ }^{239} \mathrm{Pu}$. Obviously, in this case the fuel enrichment with ${ }^{235} \mathrm{U}$ makes no difference because the averaged cross sections for ${ }^{235} \mathrm{U}$, as described above, behave in a standard way.
And finally we performed a computer estimate of the heat source density dependence $q_{T}^{f}(\vec{r}, \Phi, T, t)$ (69) on temperature for the different compositions of the uranium-plutonium fissile medium with a constant neutron flux density, presented at Figure 13. We used the dependencies presented above at Figures 10-12 for these calculations. Let us note that our preliminary calculations were made without taking into account 


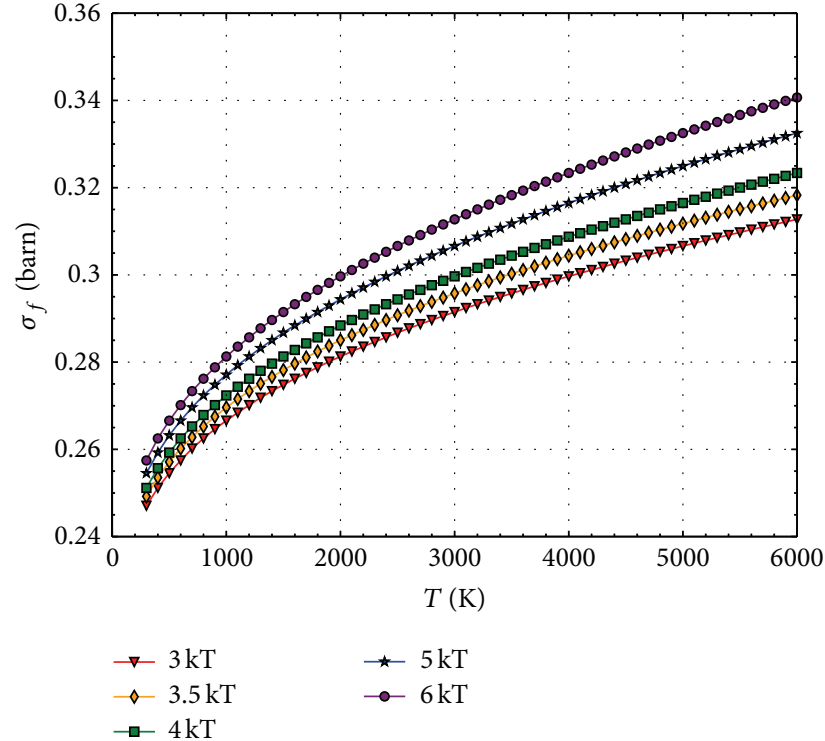

(a)

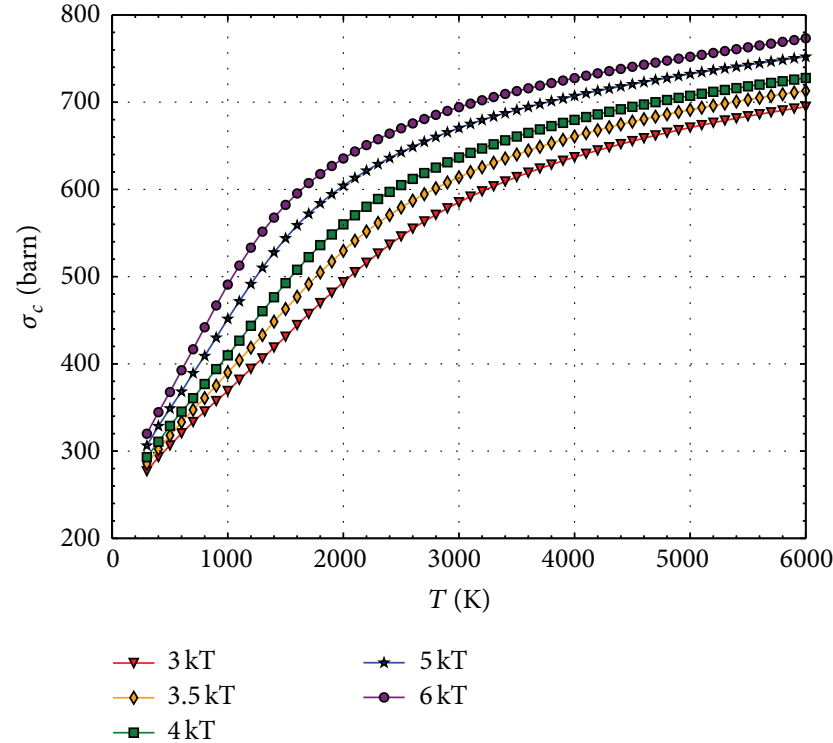

(b)

FIGURE 12: Temperature dependencies for the fission cross section (a) and radioactive capture cross section (b) for ${ }_{92}^{238} \mathrm{U}$, averaged over the combined Maxwell and Fermi spectra depending on the Maxwell and Fermi spectra joining energy and $\eta=1.8($ see $(75))$.

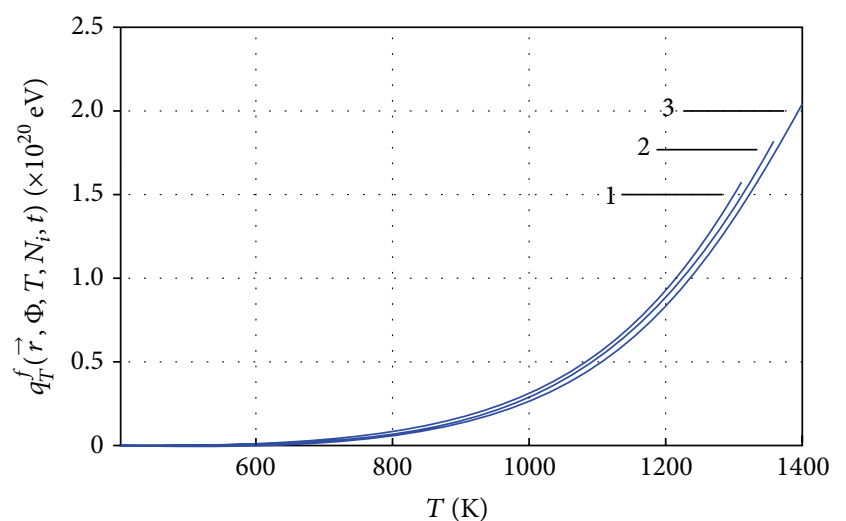

FIGURE 13: Dependence of the heat source density $q_{T}^{f}\left(r, \Phi, T, N_{i}\right.$, $t)[\mathrm{eV}]$ on the fissile medium temperature (300-1400 K) for several compositions of uranium-plutonium medium (1: $10 \% \mathrm{Pu}, 2: 5 \%$ $\mathrm{Pu}$, and 3: $1 \% \mathrm{Pu}$ ) at the constant neutron flux density $\Phi=$ $10^{13} \mathrm{n} /\left(\mathrm{cm}^{2} \cdot \mathrm{s}\right)$.

the change in the composition and density of the fissile uranium-plutonium medium, which is a direct consequence of the constant neutron flux assumption.

The necessity of such assumption is caused by the following. The reasonable description of the heat source density $q_{T}^{f}(\vec{r}, \Phi, T, t)(69)$ temperature dependence requires the solution of a system of three equations; two of them correspond to the neutron kinetics equation (flux and fluence) and to the system of equations for kinetics of the parental and child nuclides nuclear density (e.g., see $[5,16])$, while the third one corresponds to a heat transfer equation of (64) type. However, some serious difficulties arise here associated with the computational capabilities available. And here is why.
One of the principal physical peculiarities of the TWR is the fact [14] that fluctuation residuals of plutonium (or ${ }^{233} \mathrm{U}$ in Th-U cycle) over its critical concentration burn out for the time comparable with reactor lifetime of a neutron $\tau_{n}(x, t)$ (not considering the delayed neutrons) or at least comparable with the reactor period (the reactor period by definition is equal to $T(x, t)=\tau_{n}(x, t) / \rho(x, t)$, that is, a ratio of the reactor neutron lifetime to reactivity) $T(x, t)$ (considering the delayed neutrons). Meanwhile the new plutonium (or ${ }^{233} \mathrm{U}$ in $\mathrm{Th}-\mathrm{U}$ cycle) is formed in a few days (or a month) and not immediately. This means [14] that the numerical calculation must be performed with a temporal step about $10^{-6}-10^{-7}$ in case of not taking into account the delayed neutrons and $\sim 10^{-1}-10^{0}$ otherwise. At first glance, taking into account the delayed neutrons, according to [14], really "saves the day"; however, it is not always true. If the heat transfer equation contains a significantly nonlinear source, then in the case of a blowup mode, the temperature may grow extremely fast under some conditions and in 10-20 steps (with time step $10^{-6}-10^{-7} \mathrm{~s}$ ) reaches the critical amplitude that may lead to (at least) a solution stability loss or (as maximum) to a blowup bifurcation of the phase state, almost unnoticeable with a rough time step.

According to these remarks and considering the goal and format of this paper, we did not aim at finding the exact solution of some specific system of three joint equations described above. Instead, we found it important to illustrate, at the qualitative level, the consequences of the possible blowup modes in case of a nonlinear heat source presence in the heat transfer equation. As said above, we made some estimate computer calculations of the heat source density $q_{T}^{f}(\vec{r}, \Phi, T, t)(69)$ temperature dependence in 300-1400 K range for some compositions of 
uranium-plutonium fissile medium at a constant neutron flux (Figure 13).

The obtained dependencies for the heat source density $q_{T}^{f}(\vec{r}, \Phi, T, t)$ were successfully approximated by a power function of temperature with an exponent of 4 (Figure 13). In other words, we obtained a heat transfer equation with a significantly nonlinear heat source in the following form:

$$
q_{T}(T)=\text { const } \cdot T^{(1+\delta)},
$$

where $\delta>1$ in case of nonlinear thermal conductivity dependence on temperature [54-58]. The latter means that the solutions of the heat transfer equation (64) describe the so-called Kurdyumov blow-up modes [54-59], that is, such dynamic modes when one of the modeled values (e.g., temperature) turns into infinity for a finite time. As noted before, in reality, instead of reaching the infinite values, a phase transition is observed (a final phase of the parabolic temperature growth), which requires a separate model and is a basis for an entirely new problem.

Mathematical modeling of the blowup modes was performed mainly using Mathematica 5.2-6.0, Maple 10, MATLAB 7.0, utilizing multiprocessor calculations for effective application. A RungeKutta method of 8-9th order and the numerical methods of lines [65] were applied for the calculations. The numerical error estimate did not exceed $0.01 \%$. The coordinate and temporal steps were variable and chosen by the program in order to fit the given error at every step of the calculation.

Below we give the solutions for the heat transfer equation (64) with nonlinear exponential heat source (79) in uraniumplutonium fissile medium for boundary and initial parameters corresponding to the industrial reactors. The calculations were done for a cube of the fissile material with different sizes, boundary and initial temperature values. Since the temperature dependencies of the heat source density were obtained without accounting for the changing composition and density of the uranium-plutonium fissile medium, different blow-up modes can take place (HS-mode, S-mode, LSmode) depending on the ratio between the exponents of the heat conductivity and heat source temperature dependences, according to [54-59]. Therefore we considered the cases for 1st, 2nd, and 4th temperature order sources. Here the power of the source also varied by varying the proportionality factor in (79) (const $=1.00 \mathrm{~J} /\left(\mathrm{cm}^{3} \cdot \mathrm{s} \cdot \mathrm{K}\right)$ for the 1 st temperature order source; $0.10 \mathrm{~J} /\left(\mathrm{cm}^{3} \cdot \mathrm{s} \cdot \mathrm{K}^{2}\right), 0.15 \mathrm{~J} /\left(\mathrm{cm}^{3} \cdot \mathrm{s} \cdot \mathrm{K}^{2}\right)$, and $1.00 \mathrm{~J} /\left(\mathrm{cm}^{3} \cdot \mathrm{s} \cdot \mathrm{K}^{2}\right)$ for the 2 nd temperature order source; $1.00 \mathrm{~J} /\left(\mathrm{cm}^{3} \cdot \mathrm{s} \cdot \mathrm{K}^{4}\right)$ for the 4 th temperature order source).

During the calculations of the heat capacity $c_{p}$ (Figure 14(a)) and heat conductivity $\aleph$ (Figure 14(b)) of a fissile medium dependence on temperature in 300-1400 K range, the specified parameters were given by analytic expressions, obtained by approximation of experimental data for ${ }^{238} \mathrm{U}$ based on polynomial progression:

$$
\begin{aligned}
c_{p}(T) \approx & -7.206+0.64 T-0.0047 T^{2}+0.0000126 T^{3} \\
& +2.004 \cdot 10^{-8} T^{4}-1.60 \cdot 10^{-10} T^{5} \\
& -2.15 \cdot 10^{-13} T^{6}
\end{aligned}
$$

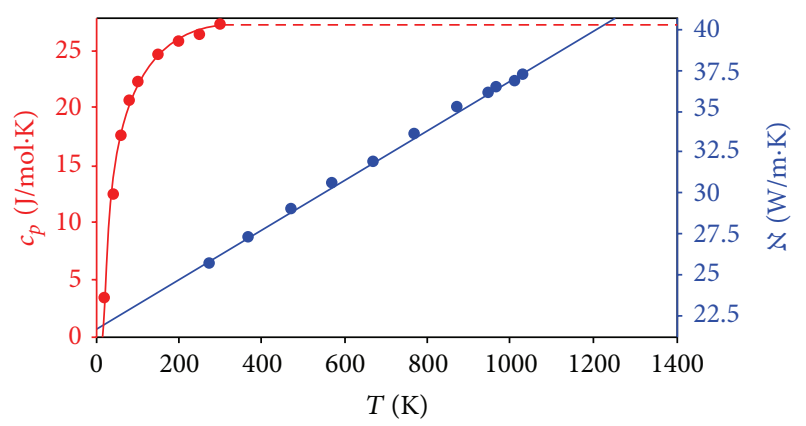

FIGURE 14: Temperature dependence of the heat capacity $c_{P}$ and heat conductivity $\chi$ of the fissile material. Points represent the experimental values for the heat capacity and heat conductivity of ${ }^{238} \mathrm{U}$.

$$
\aleph(T) \approx 21.575+0.0152661 T .
$$

And finally the heat transfer equation (64) solution was obtained for the constant heat conductivity $(27.5 \mathrm{~W} /(\mathrm{m} \cdot \mathrm{K}))$ and heat capacity $(11.5 \mathrm{~J} /(\mathrm{K} \cdot \mathrm{mol}))$, presented in Figure 15(a), and also the solutions of the heat transfer equation considering their temperature dependencies (Figures 15(b)-15(d)).

These results directly point out the possibility of the local uranium-plutonium fissile medium melting, with the melting temperature almost identical to that of ${ }^{238} \mathrm{U}$, which is $1400 \mathrm{~K}$ (Figures 14(a)-14(d)). Moreover, these regions of the local melting are not the areas of the so-called thermal peaks [66] and probably are the anomalous areas of uranium surface melting observed by Ershler and Lapteva [67] that were also mentioned in [68]. More detailed analysis of the probable temperature scenarios associated with the blowup modes is discussed below.

\section{The Blowup Modes in Neutron-Multiplying Media and the Pulse Thermonuclear TWR}

Earlier we noted the fact that due to a coolant loss at the nuclear reactors during the Fukushima nuclear accident the fuel was melted, which means that the temperature inside the active zone reached the melting temperature of uraniumoxide fuel at some moment, that is, $\sim 3000 \mathrm{~K}$.

On the other hand, we already know that the coolant loss may become a cause of the nonlinear heat source formation inside the nuclear fuel and therefore become a cause of the temperature and neutron flux blowup mode onset. A natural question arises of whether it is possible to use such blowup mode (temperature and neutron flux) for the initiation of certain controlled physical conditions under which the nuclear burning wave would regularly "experience" the so-called "controlled blow-up" mode. It is quite difficult to answer this question definitely, because such fast process has a number of important physical vagueness problems, any of which can become experimentally insurmountable for such process control.

Nevertheless such process is very elegant and beautiful from the physics point of view and therefore requires a more 


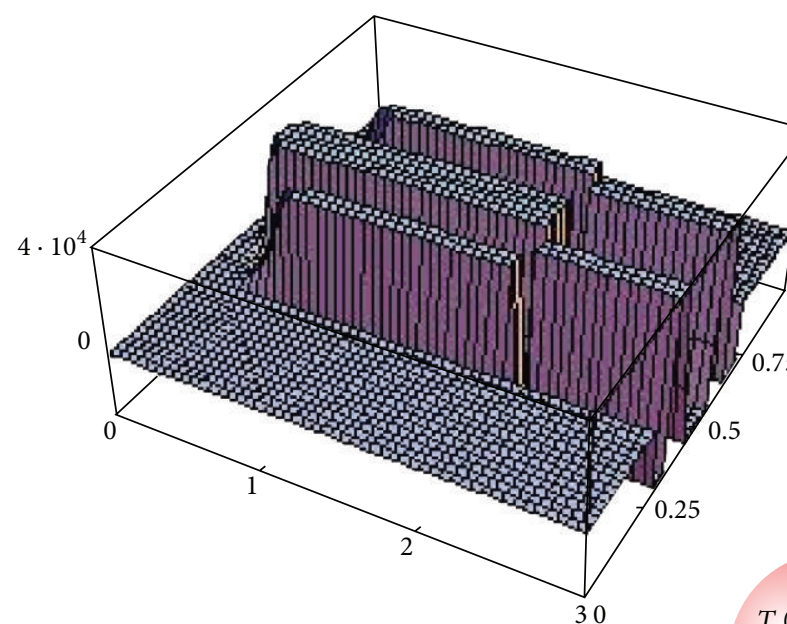

(a)

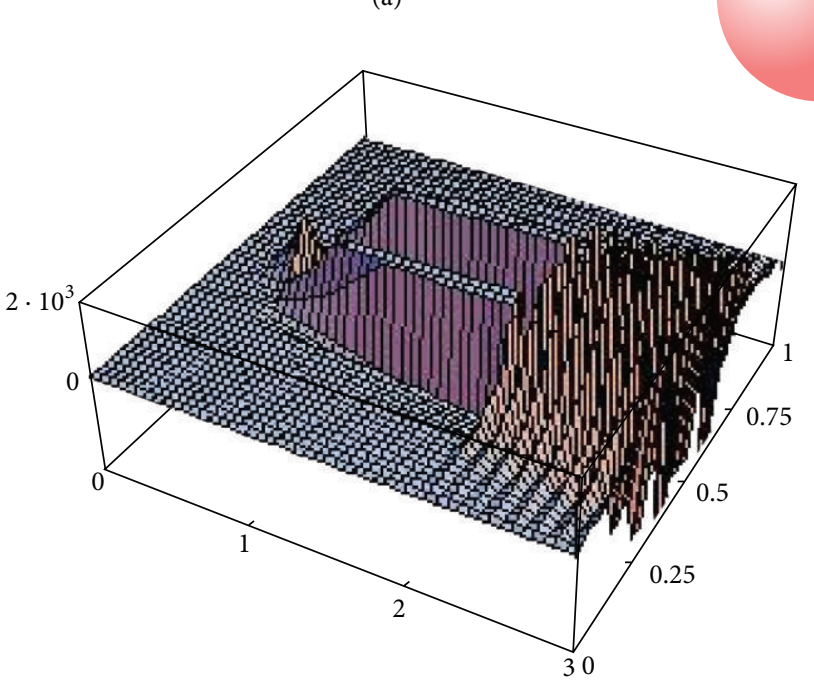

(c)

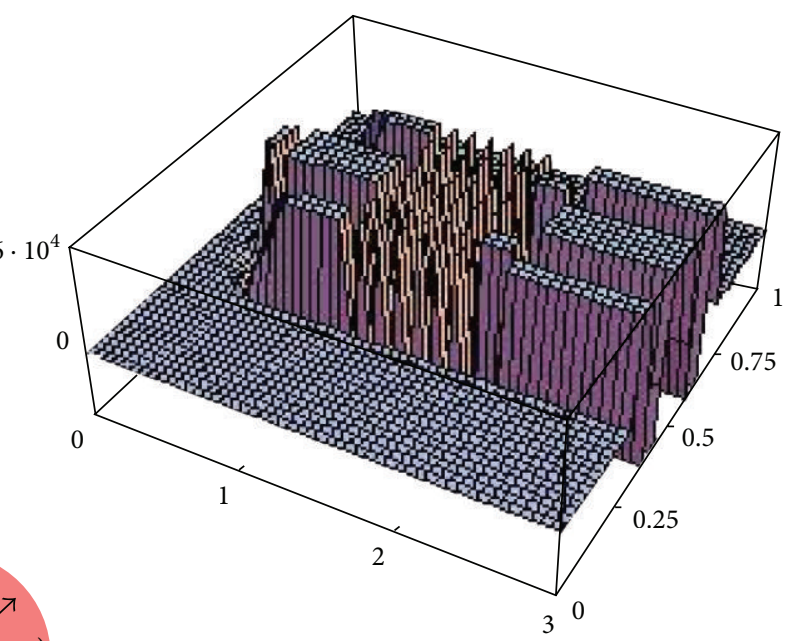

(b)

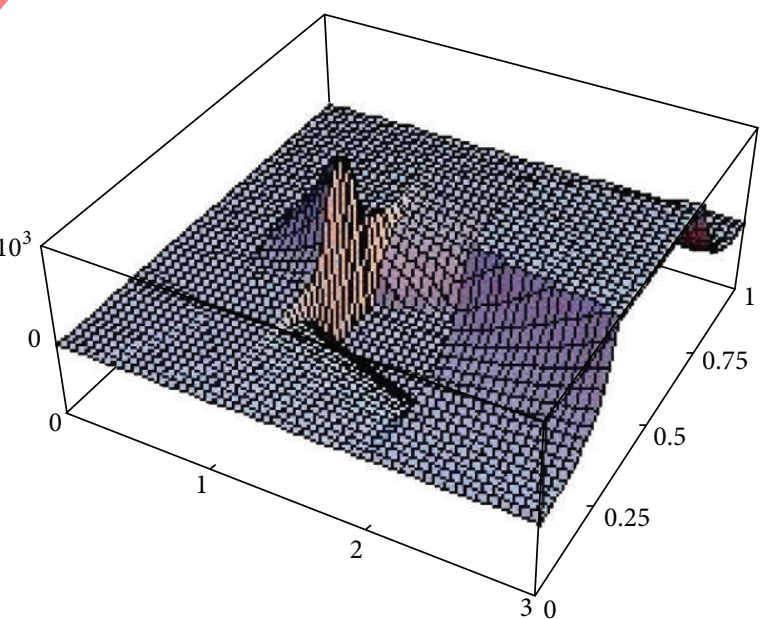

(d)

FIGURE 15: Heat transfer equation (64) solution for 3D case (crystal sizes $0.001 \times 0.001 \times 0.001 \mathrm{~mm}$, initial and boundary temperatures equal to $100 \mathrm{~K}$ ). (a) The source is proportional to the 4 th order of temperature; const $=1.00 \mathrm{~J} /\left(\mathrm{cm}^{3} \cdot \mathrm{s} \cdot \mathrm{K}^{4}\right)$; heat capacity and heat conductivity are constant and equal to $11.5 \mathrm{~J} /(\mathrm{K} \cdot \mathrm{mol})$ and $27.5 \mathrm{~W} /(\mathrm{m} \cdot \mathrm{K})$, respectively; (b) the source is proportional to the 4 th order of temperature; const = $1.00 \mathrm{~J} /\left(\mathrm{cm}^{3} \cdot \mathrm{s} \cdot \mathrm{K}^{4}\right)$; (c) the source is proportional to the 2 nd order of temperature; const $=1.00 \mathrm{~J} /\left(\mathrm{cm}^{3} \cdot \mathrm{s} \cdot \mathrm{K}^{2}\right)$; (d) the source is proportional to the 2 nd order of temperature; const $=0.10 \mathrm{~J} /\left(\mathrm{cm}^{3} \cdot \mathrm{s} \cdot \mathrm{K}^{2}\right)$. Note: in the cases $(\mathrm{b})-(\mathrm{d})$ the heat capacity and heat conductivity were determined by (80) and (81), respectively.

detailed phenomenological description. Let us try to make it in short.

As we can see from the plots of the capture and fission cross sections evolution for ${ }^{239} \mathrm{Pu}$ (Figure 10), the blowup mode may develop rapidly at $\sim 1000-2000 \mathrm{~K}$ (depending on the real value of the Fermi and Maxwell spectra joining boundary), but at the temperatures over $2500-3000 \mathrm{~K}$ the cross sections return almost to the initial values. If some effective heat sink is turned on at that point, the fuel may return to its initial temperature. However, while the blowup mode develops, the fast neutrons already penetrate to the adjacent fuel areas, where the new fissile material starts accumulating and so on (see cycles (1) and (2)). After some time the similar blowup mode starts developing in this adjacent area and everything starts over again. In other words, such hysteresis blowup mode, closely time-conjugated to a heat takeoff procedure, will appear on the background of a stationary nuclear burning wave in a form of the periodic impulse bursts.

In order to demonstrate the marvelous power of such process, we investigated the heat transfer equation with nonlinear exponential heat source in uranium-plutonium fissile medium with boundary and initial parameters emulating the heat takeoff process. In other words, we investigated the blowup modes in the Feoktistov-type uranium-plutonium reactor (1), where the temperature inside and at the boundary was deliberately fixed at $6000 \mathrm{~K}$, which corresponds to the model of the georeactor (let us note that our model georeactor is not a fast reactor; the possibility of the nuclear wave burning for a reactor other than the fast one is examined in our 
next paper [69]) [70]. Expression (75) for the neutron gas temperature, used for the calculation of the cross sections averaged over the neutron spectrum, transforms in this case to the following:

$$
T_{n} \approx\left[1+1.8 \frac{8.0 \cdot K_{2}}{\langle\xi\rangle \cdot 4.5}\right]
$$

This equation is obtained for the supposed fissile medium composition of the uranium and plutonium dicarbides [7074 ], where the ${ }^{238} \mathrm{U}$ was the major absorber (its microscopic absorption cross section for the thermalization temperatures was set at $\sigma_{a}^{8}=8.0$ barn) and the ${ }^{12} \mathrm{C}$ was the major moderator (its microscopic scattering cross section was set at $\sigma_{s}^{12}=4.5$ barn). The ${ }^{238} \mathrm{U}$ and ${ }^{12} \mathrm{C}$ nuclei concentrations ratio was set to the characteristic level for the dicarbides:

$$
K_{2}=\frac{N^{238}}{N^{12}}=0.5 .
$$

The Fermi spectrum for the neutrons in moderating and absorbing medium of the georeactor (carbon played a role of the moderator, and the ${ }^{238} \mathrm{U}$ ), ${ }^{239} \mathrm{U}$, and ${ }^{239} \mathrm{Pu}$ played the role of the absorbers was taken in the same form (76).

As an example Figure 16 shows the calculated temperature dependences of the ${ }^{235} \mathrm{U}$ and ${ }^{239} \mathrm{Pu}$ fission cross sections averaged over the neutron spectrum.

The temperature choice is conditioned by the following important consideration: "Is it possible to obtain a solution (i.e., a spatiotemporal temperature distribution) in a form of the stationary solitary wave with a limited amplitude instead of a $\delta$-function at some local spatial area, under such conditions $(6000 \mathrm{~K})$ emulating the time-conjugated heat takeoff (see Figure 10)?” As shown below, such approach really works.

Below we present some calculation characteristics and parameters. During these calculations we used the following expression for dependence of the heat conductivity coefficient:

$$
\aleph=0.18 \cdot 10^{-4} \cdot T
$$

which was obtained using the WiedemannFranz law and the data on electric conductivity of metals at temperature $6000 \mathrm{~K}$ [75]. Specific heat capacity at constant pressure was determined by value $c_{p} \approx 6 \mathrm{cal} /(\mathrm{mol} \cdot \mathrm{deg})$ according to Dulong and Petit law.

The fissile uranium-plutonium medium was modeled as a cube with dimensions $10.0 \times 10.0 \times 10.0 \mathrm{~m}$ (Figure 17). Here for heat source we used the 2 nd order temperature dependence (see (79)).

And finally Figures 17(a)-17(d) present a set of solutions of heat transfer equation (64) with nonlinear exponential heat source (79) in uranium-plutonium fissile medium with boundary and initial conditions emulating such process of heat takeoff in which initial and boundary temperatures remain constant and equal to $6000 \mathrm{~K}$.

It is important to note here that the solution set presented at Figure 17 demonstrates the solution tendency towards its

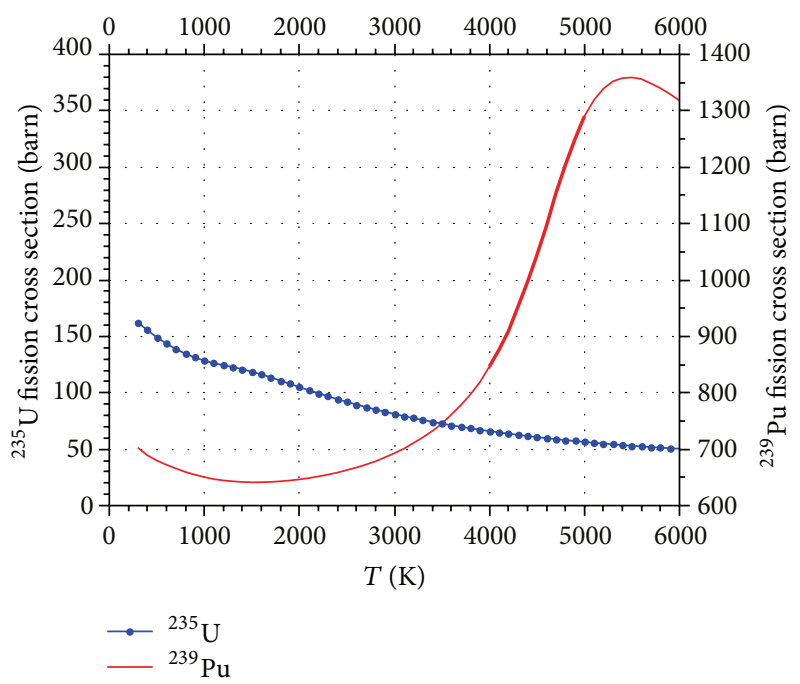

Figure 16: The temperature dependences of the ${ }^{239} \mathrm{Pu}$ fission cross section averaged over the neutron spectrum for the limit energy for the Fermi and Maxwell spectra joining equal to $3 \mathrm{kT}$. The analogous dependency for the ${ }^{235} \mathrm{U}$ is also shown.

"stationary" state quite clearly. This is achieved using the socalled "magnifying glass" approach, when the solutions of the same problem are deliberately investigated at different timescales. For example, Figure 17(a) shows the solution at the time scale $t \in\left[0,10^{-6} \mathrm{~s}\right]$, while Figure 17(b) describes the spatial solution of the problem (temperature field) for $t=$ $10^{-6} \mathrm{~s}$. Figures $17(\mathrm{c})$ and $17(\mathrm{~d})$ present the solution (spatial temperature distribution) at $t=0.5 \mathrm{~s}$ and $t=50 \mathrm{~s}$.

As one can see, the solution (Figure 17(d)) is completely identical to the previous one (Figure 17(c)), that is, the distribution established in the medium in 0.5 seconds, which allowed us to make a conclusion on the temperature field stability, starting from some moment. It is interesting that the established temperature field creates the conditions suitable for the thermonuclear synthesis reaction, that is, reaching $10^{8} \mathrm{~K}$, and such temperature field lifetime is not less than $50 \mathrm{~s}$. These conditions are highly favorable for a stable thermonuclear burning, according to a known Lawson criterion, providing the necessary nuclei concentration entering the thermonuclear synthesis reaction.

One should keep in mind, though, that the results of this section are for the purpose of demonstration only, since their accuracy is rather uncertain and requires a careful investigation with application of the necessary computational resources. Nevertheless, the qualitative peculiarities of these solutions should attract the researchers' attention to the nontrivial properties of the blowup modes, at least, with respect to the obvious problem of the inherent TWR safety violation.

\section{Conclusions}

Let us give some short conclusions stimulated by the following significant problems. 


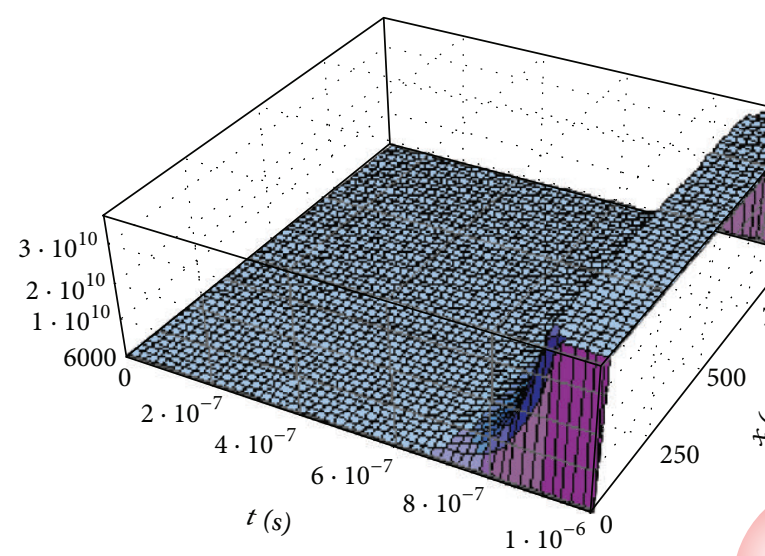

(a)

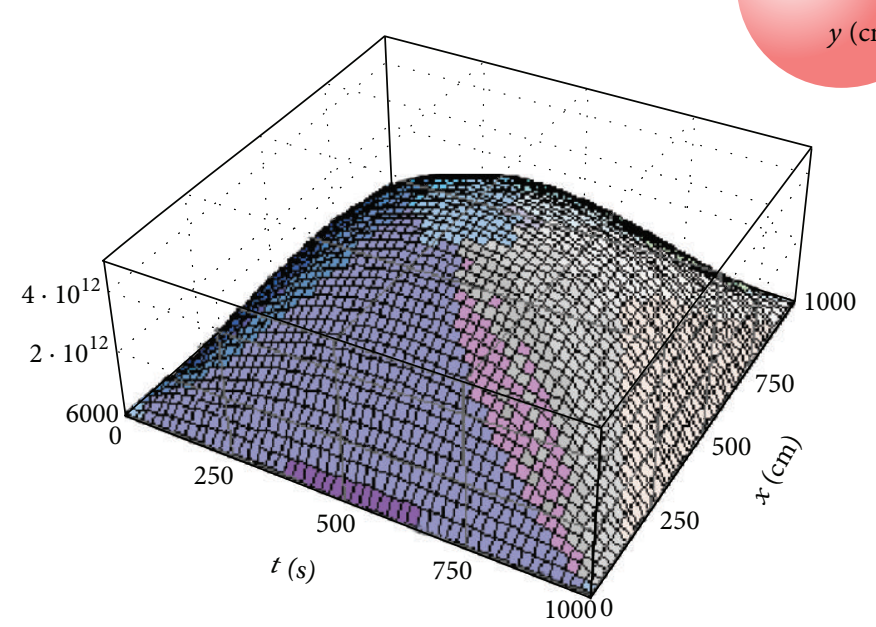

(c)

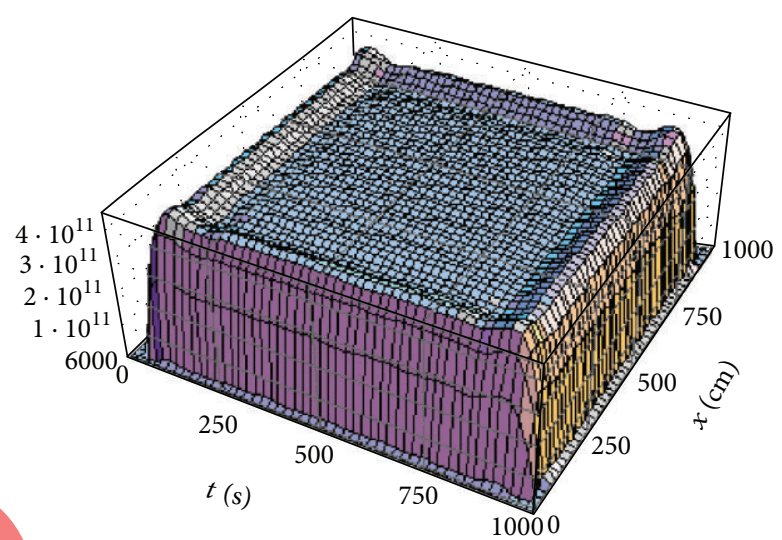

(b)

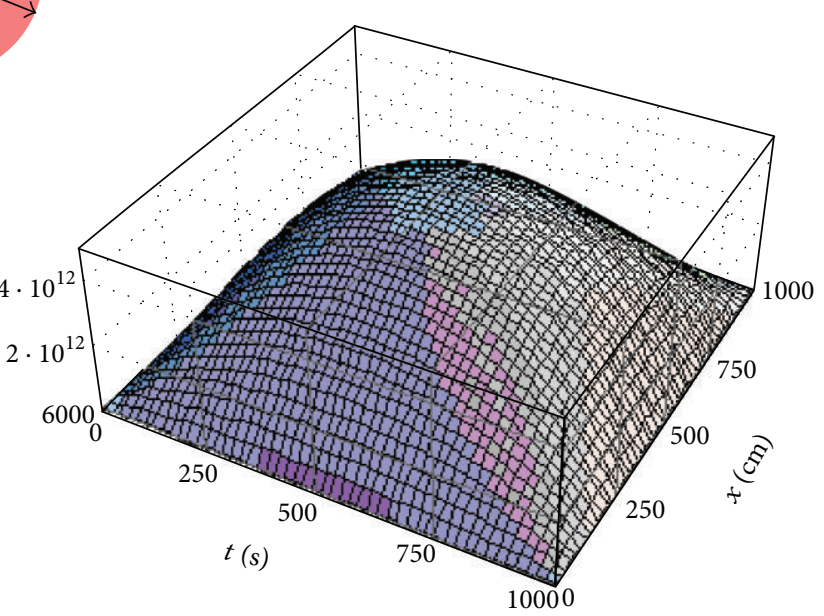

(d)

FIGURE 17: Heat transfer equation solution for a model georeactor (source $\sim$ 2nd order temperature dependence, const $=4.19 \mathrm{~J} /\left(\mathrm{cm}^{3} \cdot \mathrm{s} \cdot \mathrm{K}^{2}\right)$ ); initial and boundary temperatures equal to $6000 \mathrm{~K}$; fissile medium is a cube $10 \times 10 \times 10 \mathrm{~m}$. The presented results correspond to the following times of temperature field evolution: (a) (1-10) $10^{-7} \mathrm{~s}$, (b) $10^{-6} \mathrm{~s}$, (c) $0.5 \mathrm{~s}$, and (d) $50 \mathrm{~s}$.

(1) TWR and the Problem of dpa-Parameter in Cladding Materials. A possibility to surmount the so-called problem of dpa-parameter based on the conditions of nuclear burning wave existence in U-Pu and Th- $\mathrm{U}$ cycles is shown. In other words it is possible to find a nuclear burning wave mode, whose parameters (fluence/neutron flux, width, and speed of the wave) satisfy the dpa-condition (61) of the reactor materials radiation resistance, particularly that of the cladding materials. It can be done using the joined application of the "differential" [5] and "integral" [14-16] conditions for nuclear burning wave existence. The latter means that at the present time the problem of dpa-parameter in cladding materials in the TWR project is not an insurmountable technical problem and can be satisfactorily solved.

Here we may add that this algorithm of an optimal nuclear burning wave mode selection predetermines a satisfactory solution of other technical problems mentioned in Introduction. For example, the fuel rod length in the proposed TWR variant (see the "ideal" case in Table 1) is predetermined by the nuclear burning wave speed, which in a given case is equal to $0.254 \mathrm{~cm} /$ day $\equiv 85 \mathrm{~cm} /$ year; that is, 20 years of TWR operation requires the fuel rod length $\sim 17 \mathrm{~m}$. On the other hand, it is known [76] that for a twisted fuel rod form with two- or four-bladed symmetry, the tension emerging from the fuel rod surface cooling is $30 \%$ lower than that of a round rod with the same diameter; other conditions are equal. The same reduction effect applies to the hydraulic resistance in comparison with a round rod of the same diameter.

Another problem associated with the reactor materials swelling is also solved rather simply. It is pertinent to note that if a ferritic-martensitic material is chosen as a cladding material (Figure 6 [43]), then the swelling effect at the end of operation will be only $\sim 0.5 \%$ [43]. We could discuss other drawbacks mentioned in the introduction as well, but in our opinion, the rest of the problems are not the super obstacles for the contemporary level of nuclear engineering, as compared with the main problem of dpa-condition, and can be solved in a traditional way.

(2) The Consequences of the Anomalous ${ }^{238} U$ and ${ }^{239} \mathrm{Pu}$ Cross Sections Behavior with Temperature. It is shown that the capture and fission cross sections of ${ }^{238} \mathrm{U}$ and ${ }^{239} \mathrm{Pu}$ manifest 
a monotonous growth in $1000-3000 \mathrm{~K}$ range. Obviously, such anomalous temperature dependence of ${ }^{238} \mathrm{U}$ and ${ }^{239} \mathrm{Pu}$ cross sections changes the neutron and heat kinetics of the nuclear reactors drastically. It becomes essential to know their influence on kinetics of heat transfer because it may become the cause of a positive feedback with neutron kinetics, which may lead not only to undesirable loss of the nuclear burning wave stability but also to a reactor runaway with a subsequent disaster.

(3) Blowup Modes and the Problem of the Nuclear Burning Wave Stability. One of the causes of possible fuel temperature growth is a deliberate or spontaneous coolant loss similar to Fukushima nuclear accident. As shown above, the coolant loss may become a cause of the nonlinear heat source formation in the nuclear fuel and the corresponding mode with temperature and neutron flux blowup. In our opinion, the preliminary results of heat transfer equation with nonlinear heat source investigations point out an extremely important phenomenon of the anomalous behaviour of the heat and neutron flux blowup modes. This result poses a natural nontrivial problem of the fundamental nuclear burning wave stability and, correspondingly, of a physically reasonable application of the Lyapunov method to this problem.

It is shown that some variants of the solution stability loss are caused by anomalous nuclear fuel temperature evolution. They can lead not only to the TWR inherent safety loss, but also, through a bifurcation of states (and this is very important!), to a new stable mode when the nuclear burning wave periodically "experiences" the so-called "controlled blowup" mode. At the same time, it is noted that such fast (blowup regime) process has a number of physical uncertainties, which may happen to be experimentally insurmountable for the purposes of such process control.

(4) On-Line Remote Neutrino Diagnostics of the Intrareactor Processes. The high-power TWR or a nuclear fuel transmutation reactor is the project with the single-load, fuel burn-up, and the subsequent burial of the reactor apparatus. Hence it is necessary to perform a remote neutrino monitoring of the nuclear burning wave during the normal operation, and the neutron kinetics during the emergency situation. The details and peculiarities of the isotope composition spatiotemporal distribution calculation in the active zone of the TWR are presented in $[70,74,77]$ in detail within the inverse problem of the intrareactor processes neutrino diagnostics.

\section{Conflict of Interests}

The authors declare that there is no conflict of interests regarding the publication of this paper.

\section{References}

[1] L. Feoktistov, From Past towards the Future: From the Hopes about Bomb to Safe Reactor, RFNC-ANRISPh, Snezhinsk, Russia, 1998.

[2] S. Feinberg, "Discussion content in: Record of Proceedings Session B-10," Proceedings of the International Conference on the Peaceful Uses for Atomic Energy, Vol. 9, no. 2, p. 447,United Nations, Geneva, Switzerland, 1958.

[3] L. Feoktistov, "Neutron-fission wave," Doklady Akademii Nauk SSSR, no. 309, pp. 4-7, 1989.

[4] E. Teller, M. Ishikawa, L. Wood, R. Hyde, and J. Nuckolls, “Completely automated nuclear reactors for long-term operation II: toward a concept-level point-design of a high temperature, gascooled central power station system, part II," in Proceedings of the International Conference on Emerging Nuclear Energy Systems (ICENES '96), pp. 123-127, Lawrence Livermore National Laboratory, Obninsk, Russia, 1996.

[5] V. D. Rusov, E. P. Linnik, V. A. Tarasov et al., "Travelingwave reactor and condition of existence of nuclear burning solitonlike wave in neutron-multiplying media," Energies, vol. 4, no. 9, pp. 1337-1361, 2011.

[6] W. Seifritz, "On the burn-up theory of fast soliton reactors," International Journal of Hydrogen Energy, vol. 23, no. 2, pp. 7782, 1998.

[7] W. Seifritz, "Solitary burn-up waves in multiplying medium," Kerntechnik, vol. 65, no. 5-6, pp. 261-264, 2000.

[8] H. van Dam, "Self-stabilizing criticality waves," Annals of Nuclear Energy, vol. 27, no. 16, pp. 1505-1521, 2000.

[9] H. Sekimoto, K. Ryu, and Y. Yoshimura, "CANDLE: the new burnup strategy," Nuclear Science and Engineering, vol. 139, no. 3, pp. 306-317, 2001.

[10] N. Khizhnyak, "On the theory of the initial stage of slow nuclear burning," Problems of Atomic Science and Technology, vol. 6, pp. 279-282, 2001.

[11] X.-N. Chen and W. Maschek, "Transverse buckling effects on solitary burn-up waves," Annals of Nuclear Energy, vol. 32, no. 12, pp. 1377-1390, 2005.

[12] H. Sekimoto and Y. Udagawa, "Effects of fuel and coolant temperatures and neutron fluence on CANDLE burnup calculation," Journal of Nuclear Science and Technology, vol. 43, no. 2, pp. 189-197, 2006.

[13] V. N. Pavlovich, E. N. Khotyaintseva, V. D. Rusov, V. N. Khotyaintsev, and A. S. Yurchenko, "Reactor operating on a slow wave of nuclear fission," Atomic Energy, vol. 102, no. 3, pp. 181-189, 2007.

[14] V. Pavlovich, V. Khotyaintsev, and E. Khotyaintseva, "Physical basics of the nuclear burning wave reactor. 1," Nuclear Physics and Energetics, vol. 3, no. 2, pp. 39-48, 2008.

[15] V. Pavlovich, V. Khotyaintsev, and E. Khotyaintseva, "Physical basics of the nuclear burning wave reactor. 2. specific models," Nuclear Physics and Energetics, no. 3, pp. 39-48, 2008.

[16] V. M. Pavlovich, V. M. Khotyayintsev, and O. M. Khotyayintseva, "Nuclear burning wave reactor: wave parameter control," Nuclear Physics and Atomic Energy, vol. 11, no. 1, pp. 49-56, 2010.

[17] V. Khotyaintsev, V. Pavlovich, and E. Khotyaintseva, "Traveling wave reactor: velocity formation mechanisms," in Proceedings of the International Conference on the Physics of Reactors (PHYSOR '10): Advances in Reactor Physics to Power the Nuclear Renaissance, Pittsburgh, Pa, USA, May 2010.

[18] X.-N. Chen, W. Maschek, A. Rineiski, and E. Kiefhaber, "Solitary burn-up wave solution in a multi-group diffusionburnup coupled system," in Proceedings of the 13th International Conference on Emerging Nuclear Energy Systems (ICENES '07), pp. 236-245, Istanbul, Turkey, June 2007.

[19] X.-N. Chen, E. Kiefhaber, and W. Maschek, "Neutronic model and its solitary wave solutions for a candle reactor," in Proceedings of the 12th International Conference on Emerging Nuclear 
Energy Systems (ICENES '05), pp. 742-767, Brussels, Belgium, August 2005.

[20] Y. Ohoka, P. H. Liem, and H. Sekimoto, "Long life small candlehtgrs with thorium," Annals of Nuclear Energy, vol. 34, no. 1-2, pp. 120-129, 2007.

[21] R. Hyde, M. Ishikawa, N. Myhrvold, J. Nuckolls, and L. Wood, "Nuclear fission power for 21st century needs: enabling technologies for large-scale, low-risk, affordable nuclear electricity," Progress in Nuclear Energy, vol. 50, no. 2-6, pp. 32-91, 2008.

[22] X.-N. Chen, E. Kiefhaber, and W. Maschek, "Fundamental burn-up mode in a pebble-bed type reactor," Progress in Nuclear Energy, vol. 50, no. 2-6, pp. 219-224, 2008, Proceedings of the 2nd COE-INES International Symposium (INES-2 '06), November 26-30, 2006, Yokohama, Japan.

[23] K. D. Weaver, J. Gilleland, C. Ahlfeld, C. Whitmer, and G. Zimmerman, "A once-through fuel cycle for fast reactors," Journal of Engineering for Gas Turbines and Power, vol. 132, no. 10, Article ID 102917, 2010.

[24] W. Seifritz, "Non-linear burn-up waves in opaque neutron absorbers," Kerntechnik, vol. 60, no. 4, pp. 185-188, 1995.

[25] A. Akhiezer, D. Belozorov, F. Rofe-Beketov, L. Davydov, and Z. Spolnik, "On the theory of propagation of chain nuclear reaction in diffusion approximation," Yadernaya Fizika, vol. 62, no. 9, pp. 1567-1575, 1999.

[26] A. I. Akhiezer, D. P. Belozorov, F. S. Rofe-Beketov, L. N. Davydov, and Z. A. Spolnik, "On the theory of propagation of chain nuclear reaction," Physica A: Statistical Mechanics and its Applications, vol. 273, no. 3-4, pp. 272-285, 1999.

[27] A. Akhiezer, D. Belozorov, F. Rofe-Beketov, L. Davydov, and Z. Spolnik, "The velocity of slow nuclear burning in two-group approximation," Problems of Atomic Science and Technology, no. 6, pp. 276-278, 2001.

[28] A. Akhiezer, N. Khizhnyak, N. Shulga, V. Pilipenko, and L. Davydov, "Slow nuclear burning," Problems of Atomic Science and Technology, no. 6, pp. 272-275, 2001.

[29] G. Bartolomey, G. Bat', V. Babaykov, and M. Altukhov, Basic Theory and Methods of Nuclear Power Installations Calculation, Energoatomizdat, Moscow, Russia, 1989.

[30] W. Stacey, Nuclear Reactors Physics, John Wiley \& Sons, 2nd edition, 2007.

[31] E. Lewis, Fundamentals of Nuclear Reactors Physics, Academic Press, New York, NY, USA, 2008.

[32] W. Seifritz, "What is sustainable development? An attempt to interpret it as a soliton-like phenomenon," Chaos, Solitons and Fractals, vol. 7, no. 12, pp. 2007-2018, 1996.

[33] A. Lyapunov, The general problem of the stability of motion [Doctoral dissertation], (Russian), University of Kharkov, English translations: (1) Stability of Motion, Academic Press, New York, NY, USA, 1966.

[34] The General Problem of the Stability of Motion, A. T. Fuller translation, Taylor \& Francis, London, UK, 1992, Included is a biography by Smirnov and an extensive bibliography of Lyapunov's work, 1892.

[35] N. Chetaev, Stability of Motion, Gostekhizdat, Moscow, Russia, 1955.

[36] A. Letov, Stability of Nonlinear Control Systems, Gostekhizdat, Moscow, Russia, 1955, (Russian), English Translation by Princeton University Press, 1961.

[37] J.-J. E. Slotine and W. Li, Applied Nonlinear Control, Prentice Hall, Englewood Cliffs, NJ, USA, 1991.
[38] S. Porolo, Swelling and microstructure of the cladding steels "ei847", "ep-172" and "chs-68" after fuel elements using in bn-600 [Ph.D. thesis], 2008.

[39] V. Zelensky, I. Nekludov, and Y. Chernayaeva, Radiation Defect and Swelling of Metals, Naukova Dumka, Kiev, Ukraine, 1998.

[40] I. Akhiezer and L. Davydov, Introduction to Theoretical Radiation Physics of Metals and Alloys, Naukova dumka, Kiev, Ukraine, 1985.

[41] A. V. Kozlov, "The effect of neutron irradiation on metals under different temperatures and the opportunity of self-organization of processes occurring in them," Physics of Elementary Particles and Atomic Nuclei, vol. 37, pp. 1109-1150, 2006.

[42] G. S. Was, Fundamentals of Radiation Materials Science. Metals and Alloys, Springer, Berlin, Germany, 2007.

[43] M. Pukari and J. Wallenius, Cladding Materials and Radiation Damage, Department of Reactor Physics of Kungliga Tekniska Hogskolan, 2010.

[44] M. J. Norgett, M. T. Robinson, and I. M. Torrens, "A proposed method of calculating displacement dose rates," Nuclear Engineering and Design, vol. 33, no. 1, pp. 50-54, 1975.

[45] M. T. Robinson, "Basic physics of radiation damage production," Journal of Nuclear Materials, vol. 216, pp. 1-28, 1994.

[46] J. Lindhard, M. Scharff, and H. Schiøtt, "Range concepts and heavy ion ranges (notes on atomic collisions, II)," MatematiskeFysiske Meddelelser, Udgivet af Det Kongelige Danske Videnskabernes Selskab, vol. 33, no. 14, pp. 1-42, 1963.

[47] International Atomic Energy Agency, "51st IAEA General Conference, development of radiation resistant reactor core structural materials," Tech. Rep. NTR2007, IAEA, Vienna, Austria, 2007.

[48] J. A. Mascitti and M. Madariaga, "Method for the calculation of DPA in the reactor pressure vessel of Atucha II," Science and Technology of Nuclear Installations, vol. 2011, Article ID 534689, 6 pages, 2011.

[49] S. Fomin, Y. Melnik, V. Pilipenko, and N. Shulga, "Self-sustained regime of nuclear burning wave in $\mathrm{u}$-pu fast neutron reactor with pb-ni coolant," Problems in Atomic Science and Technology, vol. 3, pp. 156-163, 2007.

[50] S. Fomin, Y. Mel'nik, V. Pilipenko, and N. Shul'ga, "Investigation of self-organization of the non-linear nuclear burning regime in fast neutron reactors," Annals of Nuclear Energy, vol. 32, no. 13, pp. 1435-1456, 2005.

[51] Y. Melnik, V. Pilipenko, A. Fomin, S. Fomin, and N. Shulga, "Study of a self-regulated nuclear burn wave regime in a fast reactor based on a thorium-uranium cycle," Atomic Energy, vol. 107, pp. 49-56, 2009.

[52] S. Shirokov, Nuclear Reactor Physics, Naukova dumka, Kiev, Russia, 1992, (Russian).

[53] V. Ukraintsev, Reactivity Effects in Energetic Installations, Handbook, Obninsk Institute for Nuclear Power Engineering, Obninsk, Russia, 2000, (Russian).

[54] T. Akhromeeva, S. Kurdyumov, G. Malinetskii, and A. Samarskii, Non-Stationary Structures and Diffusive Chaos, Nauka, Moscow, Russia, 1992, (Russian).

[55] A. A. Samarskii, V. A. Galaktionov, S. P. Kurdyumov, and A. P. Mikhailov, Blow-Up in Quasilinear Parabolic Equations, Walter de Gruyter, Berlin, Germany, 1995.

[56] S. P. Kurdyumov, Blow-up Modes. Evolution of the Idea. The Laws of Co-Evolution of Complex Systems, Nauka, Moscow, Russia, 1999. 
[57] S. Kurdyumov, Blow-Up Modes, Fizmatlit, Moscow, Russia, 2006.

[58] E. Knyazeva and S. Kurdyumov, Synergetics: Nonlinearity of Time and Landscape of Co Evolution, KomKniga, Moscow, Russia, 2007.

[59] V. Rusov, V. Tarasov, and S. Chernegenko, "Blow-up modes in uranium-plutonium fissile medium in technical nuclear reactors and georeactor," Problems of Atomic Science and Technology, vol. 97, pp. 123-131, 2011 (Russian).

[60] D. Skorov, Y. Bychkov, and A. Dashkovskii, Reactor Material Science, Atomizdat, Moscow, Russia, 1979, (Russian).

[61] B. Nadykto, Ed., Plutonium. Fundamental problems, RFNCAREPRI, Sarov, Russia, 2003, (Russian).

[62] Los Alamos National Laboratory, ENSDF/B-VI, 1998.

[63] N. Fedorov, Short Reference Book for Engineer-Physicist. Nuclear Physics and Atomic Physics, State Publishing Company for Atomic Science and Technology Literature, Moscow, Russia, 1961, (Russian).

[64] V. Vladimirov, Practical Problems on Nuclear Reactors Operation, Energoatomizdat, Moscow, Russia, 1986, (Russian).

[65] A. Samarskii and A. Gulin, Numerical Methods in Mathematical Physics, Nauchnyi mir, Moscow, Russia, 2003, (Russian).

[66] G. H. Kinchin and R. S. Pease, "The displacement of atoms in solids by radiation," Reports on Progress in Physics, vol. 18, no. 1, article 301, pp. 590-615, 1955.

[67] B. V. Ershler and F. S. Lapteva, "The evaporation of metals by fission fragments," Journal of Nuclear Energy (1954), vol. 4, no. 4, pp. 471-474, 1957.

[68] I. M. Lifshits, M. I. Kaganov, and L. V. Tanatarov, "On the theory of the changes produced in metals by radiation," The Soviet Journal of Atomic Energy, vol. 6, no. 4, pp. 261-270, 1960.

[69] V. Rusov, V. Tarasov, M. Eingorn, S. Chernezhenko, and A. Kakaev, "Ultraslow wave nuclear burning of uraniumplutonium fissile medium on epithermal neutrons," In preparation, http://xxx.tau.ac.il/abs/1409.7343.

[70] V. D. Rusov, V. N. Pavlovich, V. N. Vaschenko et al., "Geoantineutrino spectrum and slow nuclear burning on the boundary of the liquid and solid phases of the Earth's core," Journal of Geophysical Research B: Solid Earth, vol. 112, no. 9, Article ID B09203, 2007.

[71] V. F. Anisichkin, A. P. Ershov, A. Bezborodov et al., "The possible modes of chain nuclear reactions in the Earth's core," in VII Zababa Khin's Sientific Lectures, 2003.

[72] V. Anisichkin, A. Bezborodov, and I. Suslov, "Chain fission reactions of nuclides in the earths core during billions years," Atomic Energy, vol. 98, pp. 370-379, 2005.

[73] V. F. Anisichkin, "Do the planets explode?" Burning and Explosion Physics, vol. 33, no. 1, pp. 138-142, 1997 (Russian).

[74] V. Rusov, V. Tarasov, and D. Litvinov, Reactor Antineutrino Physics, URSS, Moscow, Russia, 2008 (Russian).

[75] V. Zharkov, The Inner Structure of Earth and Planets, Nauka, Moscow, Russia, 1983.

[76] A. G. Lanin and I. I. Fedik, "Selecting and using materials for a nuclear rocket engine reactor," Physics-Uspekhi, vol. 54, no. 3, pp. 305-318, 2011.

[77] V. D. Rusov, T. N. Zelentsova, V. A. Tarasov, and D. A. Litvinov, "Inverse problem of remote neutrino diagnostics of intrareactor processes," Journal of Applied Physics, vol. 96, no. 3, pp. 17341739, 2004. 


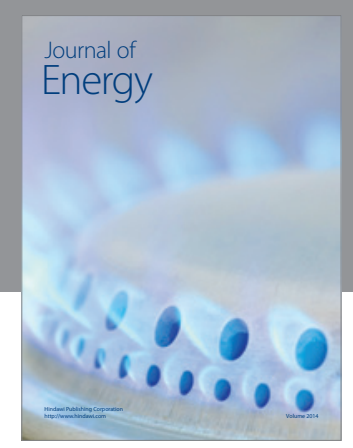

Journal of

Industrial Engineering
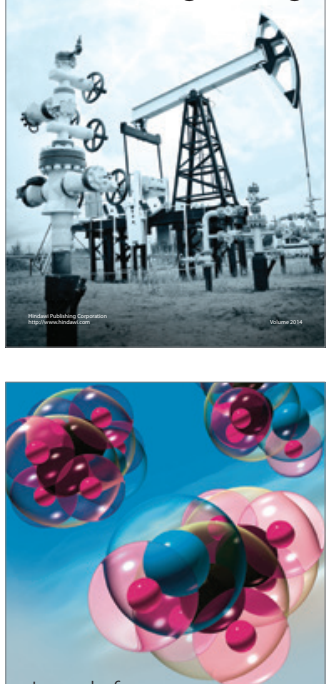

Fuels
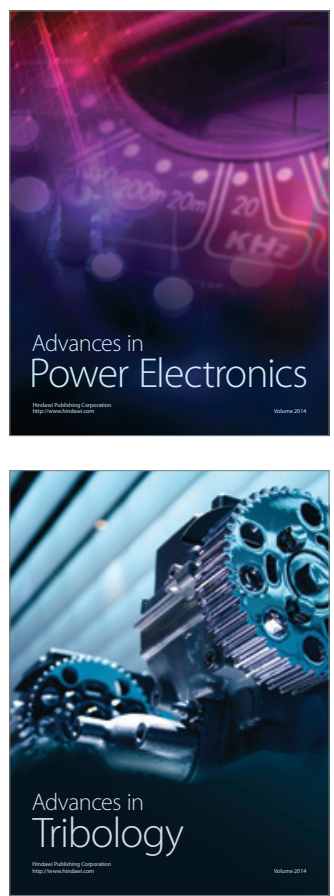

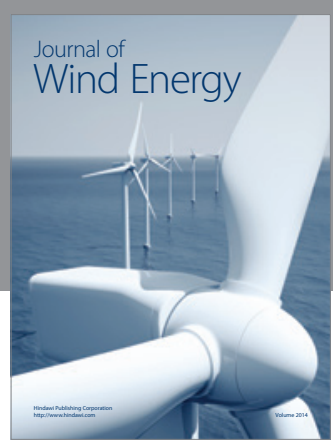

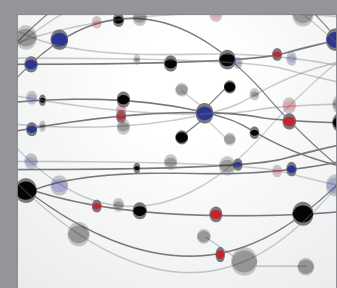

The Scientific World Journal

Submit your manuscripts at http://www.hindawi.com

Journal of

Structures
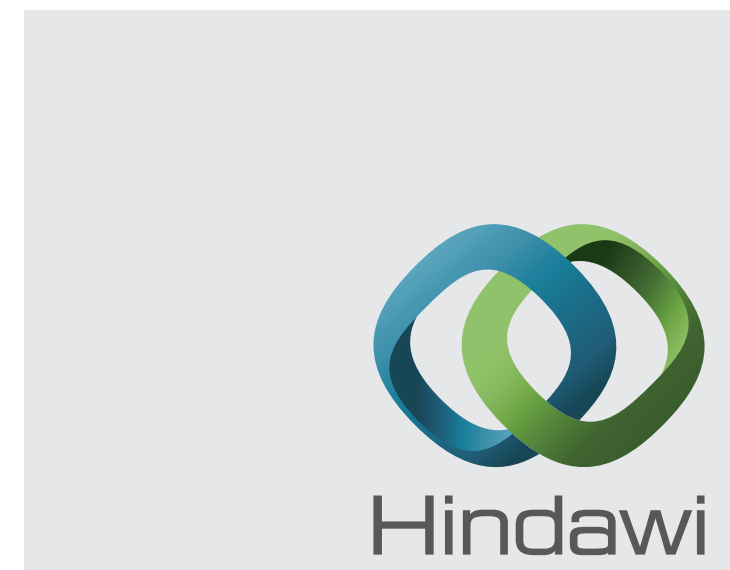

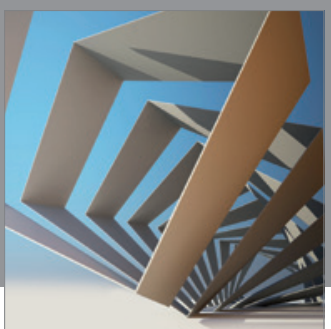

Rotating

Machinery
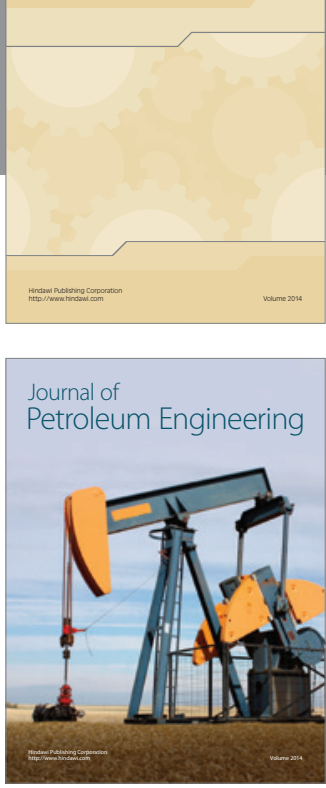

Journal of

Solar Energy
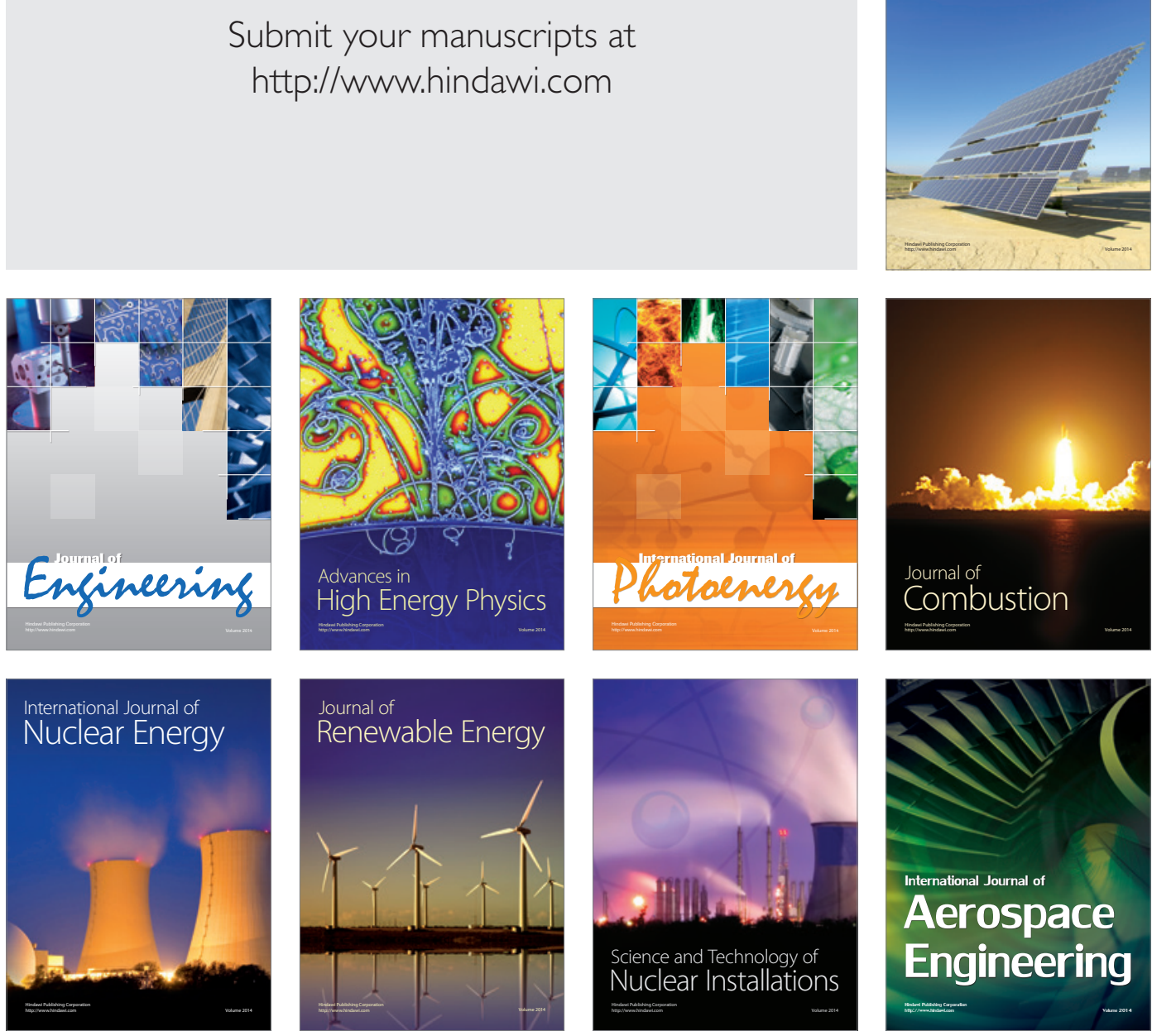